\title{
Antibiotika und der Gastrointestinaltrakt
}

\author{
Christoph Lübbert
}

\begin{tabular}{|l|l|}
\hline Übersicht & \\
\hline Einleitung & 161 \\
\hline Was sind Antibiotika? & 161 \\
\hline $\begin{array}{l}\text { Auswirkungen auf das Mikrobiom } \\
\text { Wie werden Antibiotika sinnvoll }\end{array}$ & 162 \\
\hline $\begin{array}{l}\text { eingesetzt? } \\
\text { Resistenz und Multiresistenz }\end{array}$ & 163 \\
\hline $\begin{array}{l}\text { Krankheitsbilder und ihre } \\
\text { antibiotische Therapie }\end{array}$ & 163 \\
\hline
\end{tabular}

\section{Einleitung}

GI-Trakt. Der Gastrointestinaltrakt ist mit einer Schleimhautoberfläche von ca. $300 \mathrm{~m}^{2}$ (der Fläche eines Tennisplatzes entsprechend) die größte Kontaktfläche zwischen dem Körper und der Umwelt [1]. Dabei kommt es unvermeidlich zum Aufeinandertreffen mit potenziell pathogenen Erregern bzw. Toxinen. Aufgrund des niedrigen pH-Wertes im Magen sind Infektionen in diesem Organ trotz der exponierten Stellung vergleichsweise selten. Geringere Säurekonzentrationen im Dünn- und Dickdarm ermöglichen das Überleben sowohl einer physiologischen Darmflora als auch pathogener Erreger. Aufgrund der deutlich längeren Passagezeit ist die Bakteriendichte im Kolon ( $10^{12}$ koloniebildende Einheiten [KBE] pro Gramm Feuchtmasse) um ein Vielfaches höher als im Jejunum $\left(10^{4}\right.$ $\mathrm{KBE} / \mathrm{g}$ Feuchtmasse) oder im Ileum $\left(10^{6} \mathrm{KBE} / \mathrm{g}\right.$ Feuchtmasse) [1].

Infektionen im GI-Trakt. Infektiöse Diarrhöen zählen zu den häufigsten Erkrankungen weltweit und sind nach Angaben der Weltgesundheitsorganisation WHO eine der 5 häufigsten Todesursachen [2]. Ursächlich kommen virale, bakterielle und parasitäre Erreger in Betracht. Sind Magen und Dünndarm von einer Entzündung betroffen, entsteht eine Gastroenteritis. Ist nur der Dünndarm befallen, wird von einer Enteritis gesprochen, bei alleinigem Dickdarmbefall von einer Kolitis. Die Kombination aus Dünndarm- und Dick- darmbefall wird als Enterokolitis bezeichnet. Die Therapie bakterieller Infektionen des GI-Trakts erfolgt in der Regel mit Antibiotika.

\section{Was sind Antibiotika?}

Antibiotika zählen zu den wichtigsten Arzneimitteln zur Behandlung bakterieller Infektionskrankheiten. Im engeren Sinne handelt es sich dabei um einen Sammelbegriff für bestimmte Stoffwechselprodukte von Pilzen oder Bakterien, die in der Lage sind, das Wachstum von anderen Mikroorganismen zu hemmen oder diese abzutöten. Gegen Viren sind Antibiotika wirkungslos.

Erste Antibiotika. Die Entdeckung des Schimmelpilzes Penicillium chrysogenum als „Bakterienkiller“ im Jahr 1928 war ein medizinischer Meilenstein und sicherte dem schottischen Bakteriologen Alexander Fleming 1945 den Nobelpreis für Medizin. Penicillin - das Stoffwechselprodukt des Schimmelpilzes - gilt seitdem als das Synonym für Antibiotika schlechthin. Seit der Markteinführung des Penicillins in den 1940er Jahren haben Antibiotika Millionen von Menschen das Leben gerettet.

Als erstes am Menschen angewandtes Antibiotikum gilt heute allerdings nicht das Penicillin, sondern das von Paul Ehrlich entdeckte Arsphenamin, das ab 1910 von 
der deutschen Firma Hoechst produziert wurde und als Salvarsan in den Handel kam. Es ermöglichte erstmals eine wirksame und relativ ungefährliche Therapie der damals weitverbreiteten Syphilis. Ansonsten war das Wirkungsspektrum von Salvarsan jedoch begrenzt, weshalb es in der modernen Medizin von neueren Wirkstoffen abgelöst wurde.

Grundsätzlich wird zwischen natürlichen und künstlich hergestellten Antibiotika unterschieden. Neben den natürlich erzeugten Wirkstoffen können Antibiotika auch vollsynthetisch gewonnen werden.

Wirkmechanismen. Antibiotika, die das Wachstum von Mikroorganismen zu hemmen vermögen, fallen in die Gruppe der bakteriostatisch wirkenden Substanzen. Dazu zählen z. B. Sulfonamide, Tetracycline, Chloramphenicol, Makrolide oder Lincosamide. Antibiotika, die in der Lage sind, Mikroorganismen abzutöten, verfügen über eine bakterizide Wirkung, z. B. Penicilline, Cephalosporine, Carbapeneme, Rifamycine, Lipopeptide oder Polymyxine.

Wichtige biochemische Wirkungsmechanismen sind Hemmung der bakteriellen Zellwandsynthese, Beeinflussung der Zellmembran, Hemmung der bakteriellen Proteinbiosynthese, Antimetabolitenwirkung und Hemmung der bakteriellen DNA- bzw. RNA-Synthese. Antibiotika sind in der Regel so aufgebaut, dass sie nach dem Schlüssel-Schloss-Prinzip nur an Bakterienzellen, nicht aber an menschliche oder tierische Zellen „andocken“ können. So wird eine-im Gegensatz zu den meisten Antimykotika - gezielte Beeinträchtigung oder Zerstörung bakterieller Zellen ermöglicht.

Anwendung. Die Anwendung erfolgt meist peroral oder intravenös, seltener intramuskulär. Allerdings sind einige Substanzen auch zur topischen Behandlung

Abb. 1 Dynamik des Resistenzproblems im Krankenhausbereich. oberflächlicher Infektionen der Haut und Schleimhäute zugelassen (z.B. Fusidinsäure) oder sind ganz auf diesen Anwendungsbereich beschränkt. Weltweit sind antibiotische Präparate mit 10 - 15\% Marktanteil die am häufigsten verschriebenen Medikamente [3].

Noch heute gibt es bei schwerwiegenden bakteriellen Infektionen kaum eine ernstzunehmende Alternative zum Einsatz von Antibiotika.

\section{Auswirkungen auf das Mikrobiom}

Die normale Mikroflora von Haut und Schleimhäuten wirkt als Barriere gegen eine Kolonisierung durch potenziell pathogene Mikroorganismen und gegen übermäßiges Wachstum von opportunistischen Mikroorganismen. Diese physiologische Kontrolle des Wachstums von opportunistischen Mikroorganismen wird als Kolonisationsresistenz bezeichnet [4].

Störungen der Mikroflora. Die Verabreichung von antimikrobiell wirksamen Substanzen - egal ob therapeutisch oder prophylaktisch - verursacht klinisch relevante Störungen des ökologischen Gleichgewichtes zwischen dem Wirt und der normalen Mikroflora, insbesondere im Bereich der Darmflora [4]. Alterationen der physiologischen Darmflora entwickeln sich in Abhängigkeit von den biologischen Eigenschaften des eingesetzten Antibiotikums (Pharmakodynamik), dessen Absorption und Elimination sowie möglicher enzymatischer Inaktivierung und/oder Bindung an den Fäkalinhalt. Die häufigsten Störungen sind Durchfall (antibiotikaassoziierte Diarrhö, AAD) und Überwucherung durch Pilze (insbesondere Candida-Spezies), die üblicherweise nach dem Ende der Behandlung enden.

Erhaltung der normalen Mikroflora. Eine ausgewogen zusammengesetzte intestinale Mikroflora bietet einen Schutz gegen die Etablierung resistenter Bakterien [4]. Durch die Anwendung von antimikrobiellen Substanzen mit schmalem Wirkspektrum, die zu keiner gravierenden Störung der Kolonisationsresistenz führen, lässt sich das Risiko der Entstehung und Übertragung von resistenten Bakterienstämmen zwischen Patienten und die Ausbreitung von resistenten Klonen unter den Mikroorganismen deutlich reduzieren (Abb.1). 
Der Einsatz von Antibiotika mit schmalem Wirkspektrum reduziert Störungen der normalen Mikroflora und damit das Risiko der Entstehung resistenter Bakterienstämme.

\section{Wie werden Antibiotika sinnvoll eingesetzt?}

Der Nutzen einer empirischen Antibiotikatherapie bei bakteriellen Infektionen des Gastrointestinaltrakts, die primär mit Durchfall einhergehen (infektiöse Diarrhö), ist umstritten [5]. Während mit dem schlecht resorbierbaren Fluorchinolon-Antibiotikum Norfloxacin bei der Campylobacter-Enteritis eine geringe Reduktion der Krankheitsdauer erreicht werden konnte, blieb die antimikrobielle Therapie bei Patienten mit Salmonellose ohne nachweisbaren Effekt [6]. Zudem müssen mögliche unerwünschte Wirkungen einer Antibiotikatherapie berücksichtigt werden wie erhöhtes Risiko für ein hämolytisch-urämisches Syndrom (HUS) bei EHECInfektion, erhöhte Rate an Dauerausscheidern bei Salmonellose und erhöhtes Risiko für das Auftreten einer Clostridium-difficile-Infektion (CDI) [1].

Empirische Therapie bei infektiöser Diarrhö. Eine empirische Antibiotikatherapie kann bei schwer erkrankten Patienten mit einer hohen Stuhlfrequenz (>8/d), Immundefizienz, hohem Alter oder signifikanter Komorbidität erwogen werden. Mittel der Wahl ist bislang Ciprofloxacin [1]. Allerdings sollte die zunehmende Inzidenz von Infektionen mit Ciprofloxacinresistenten Erregern, insbesondere CampylobacterSpezies, berücksichtigt werden. Eine Alternative ist Azithromycin (1-mal 500 mg p.o. für 3 Tage) [1]. Auch eine Einmalgabe von Ciprofloxacin, Levofloxacin oder Azithromycin hat sich in der empirischen antibiotischen Therapie der nicht invasiven akuten Diarrhö prinzipiell als wirksam erwiesen [7]. Bei leichteren Verlaufsformen kommt auch das nicht resorbierbare Antibiotikum Rifaximin in einer Dosis von 2-bis 3-mal $400 \mathrm{mg}$ p.o. in Betracht [8].

Gezielte Therapie. Die gezielte Therapie von schweren infektiös bedingten Durchfallerkrankungen setzt eine differenzierte mikrobiologische Stuhluntersuchung voraus. Antibiotikaresistenzen - gerade bei importierten Fällen (vor allem aus Indien) - sind in zunehmendem Maße zu berücksichtigen [9].
Problem Resistenzentwicklung. Allgemein gilt, dass Infektionen des GI-Trakts sowie seiner „Anhangsorgane“ einen wesentlichen Teil des resistenten Erregerspektrums ausmachen, sodass die Wahl des richtigen Antibiotikums in der empirischen Therapie schwieriger wird. Adäquate mikrobiologische Diagnostik als Grundlage für richtige Therapieentscheidungen wird trotz des nicht immer einfachen Zugangsweges für die Gewinnung des Probenmaterials immer wichtiger [10]. Wichtigste Risikofaktoren für eine Infektion mit resistenten oder multiresistenten Erregern sind nosokomiale bzw. „nosokommunale“ Akquisition sowie eine Antibiotikavortherapie (Abb.1). Diese Faktoren müssen bei der Initiierung einer empirischen antimikrobiellen Behandlung in Betracht gezogen werden. Zeitverzögerungen beim „Greifen“ der Therapie korrelieren mit einer Exzess-Letalität [11].

Eine empirische Antibiotikatherapie bei bakterieller Diarrhö ist umstritten. Bei zunehmender Resistenzentwicklung wird eine adäquate mikrobiologische Diagnostik als Grundlage einer gezielten Therapie immer wichtiger.

\section{Resistenz und Multiresistenz}

Resistenzmechanismen. Durch die häufige Verschreibung und den teilweise übertriebenen Einsatz von Antibiotika in der Humanmedizin, aber auch wegen des exzessiven Einsatzes von Antibiotika in der industriellen Tierproduktion durch Veterinärmediziner haben Bakterien eine ganze Reihe von Resistenzmechanismen gegen Antibiotika entwickelt: So können viele Bakterienspezies Antibiotika enzymatisch abbauen (z. B. durch Beta-Laktamasen) oder so umgestalten, dass sie biologisch unwirksam werden. Andere verändern ihre Zielstrukturen (Rezeptoren), an denen Antibiotika angreifen können, sodass der „Schlüssel“ nicht mehr zum „Schloss“ passt. Außerdem können Antibiotika aktiv aus Zellen z. B. mittels Effluxpumpen ausgeschleust werden oder ihre Aufnahme in die Zelle wird erschwert (z.B. durch den Verlust von Porinen in der äußeren Zellmembran) (Abb. 2).

Entwicklung multiresistenter Erreger. Die rasche Veränderung von Bakterien durch Mutation stellt aktuell eines der größten Probleme in der Medizin dar. Durch die Aufnahme von Antibiotika bereits über Umwelt und Nahrungskette (insbesondere Fleisch), durch den ungerechtfertigten Einsatz von Substanzen mit extrem breitem Wirkspektrum und durch einen häufig zu langen „prophylaktischen“ Antibiotikaeinsatz, z. B. im 


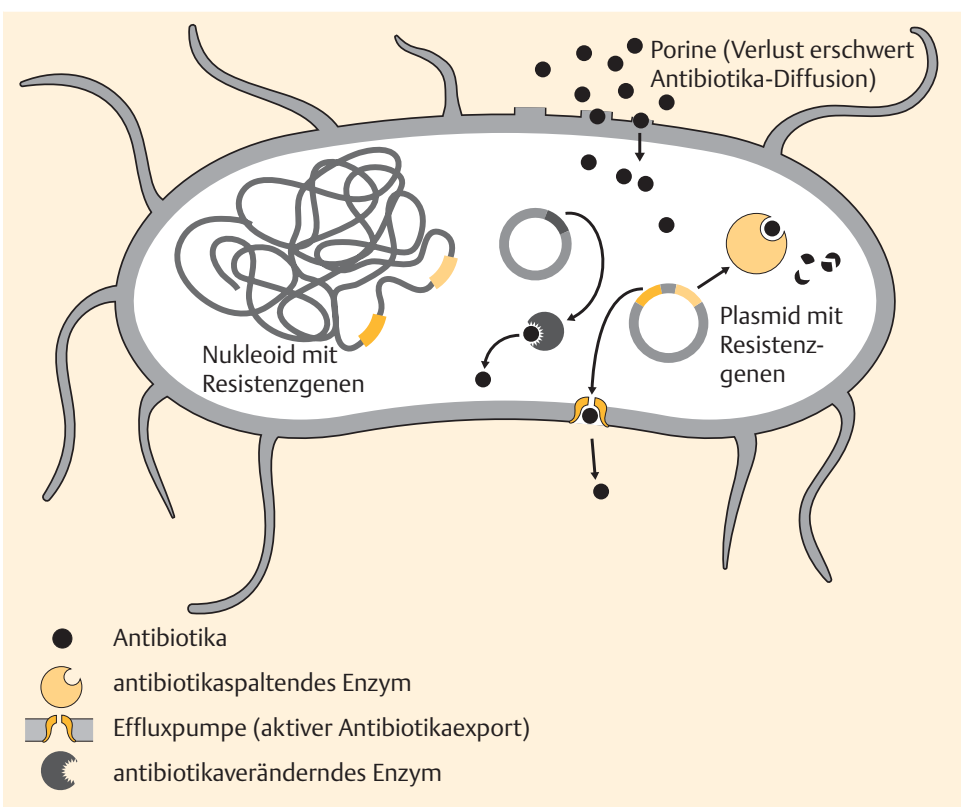

Abb. 2 Typische Resistenzmechanismen bei Bakterien.

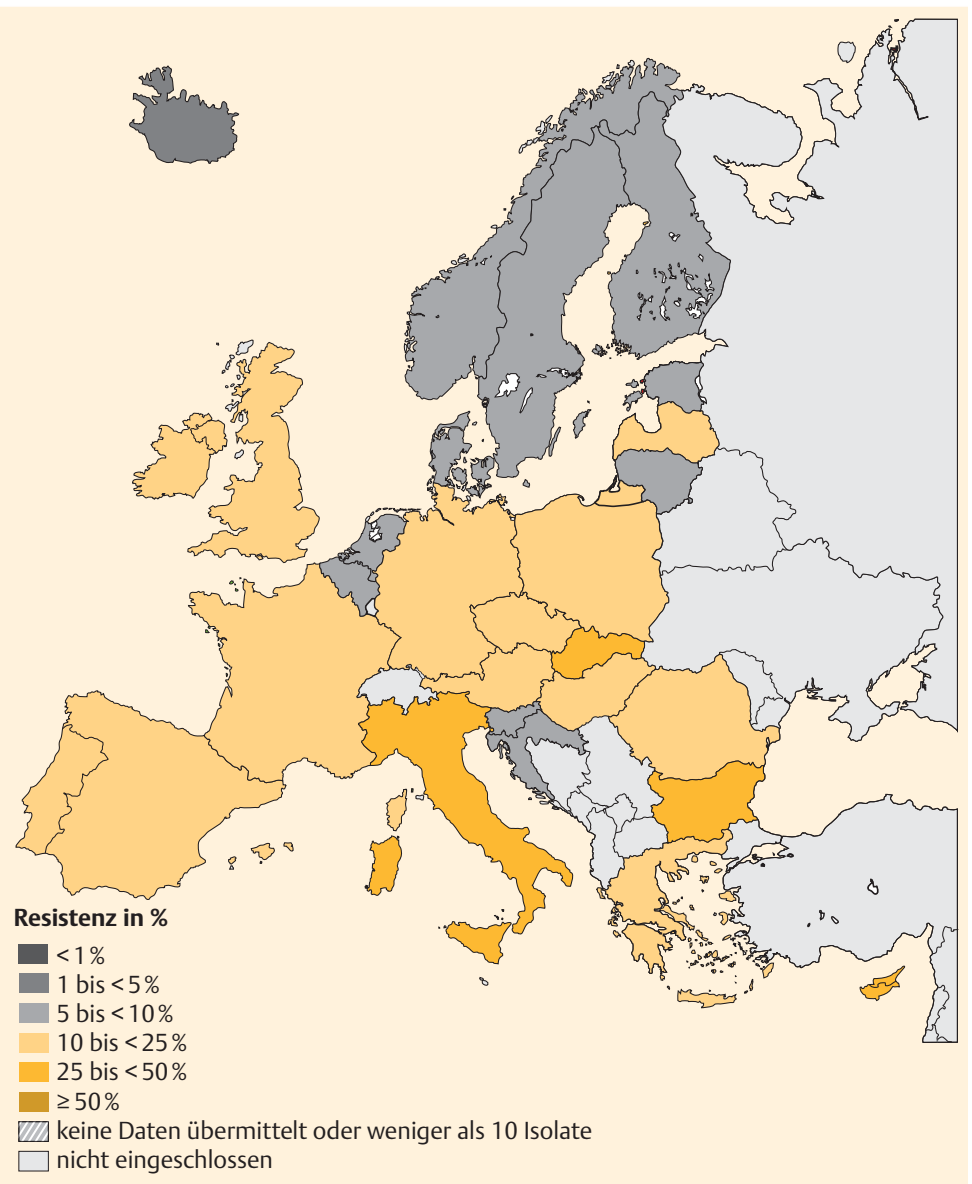

Abb.3 Anteil der E.-coli-Isolate mit Resistenz gegenüber Cephalosporinen der 3. Generation in Europa (ECDC 2015).
Rahmen chirurgischer Eingriffe, schreitet die Resistenzentwicklung stetig weiter voran. In den Abb. 3 und Abb. 4 sind beispielhaft wichtige Resistenzentwicklungen auf europäischer Ebene bei wichtigen gramnegativen Erregern mit Multiresistenz (MRGN) dargestellt.

Situation in Deutschland. Im europäischen Vergleich liegt Deutschland beim Gesamtverbrauch an Antibiotika im unteren Drittel und damit eher bei den moderaten Verbrauchsländern [12] (Abb.5). Eine Besonderheit in den Verbrauchszahlen bilden saisonale Schwankungen (Erkältungszeit im Winter). Der (sinnlose) Einsatz von Antibiotika bei Erkältungskrankheiten, die vorwiegend durch virale Erreger ausgelöst werden, dürfte demnach überall in Europa eine Rolle spielen [12].

Zur Resistenzproblematik hinzu kommt mangelnde Hygiene in Pflegeheimen und Krankenhäusern. Gerade Großkrankenhäuser bieten Bakterien ein äußerst effektives „Überlebenstraining“, bei dem diese lernen, sich perfekt an den durch Antibiotika ausgeübten Selektionsdruck anzupassen. Dabei neu gewonnene genetische Informationen tauschen Bakterienstämme z.B. über Plasmide untereinander aus, sodass in der Folge opportunistisch agierende multiresistente Erregerstämme entstehen, die insbesondere abwehrgeschwächte Patienten kolonisieren und infizieren. Aktuelle Zahlen zum Phänomen der Multiresistenz sind durchaus alarmierend: In Deutschland sterben laut ARS (Antibiotika-Resistenz-Surveillance) jährlich bis zu 30000 Menschen an Infektionen durch resistente Erreger, außerdem steigt die Zahl der Krankenhausinfektionen weiter an (letzter Stand der Schätzungen ca. 600000 Fälle) [13].

Zu häufiger Einsatz, falsche Anwendung von Antibiotika mit extrem breitem Wirkspektrum, Aufnahme über die Nahrungskette sowie mangelnde Hygiene in Kliniken lassen das Phänomen Multiresistenz alarmierend zunehmen.

\section{Krankheitsbilder und ihre antibiotische Therapie}

\section{Akute infektiöse Diarrhöen}

Die aktuelle Epidemiologie der wichtigsten Enteropathogene in Deutschland ist in Tab. 1 dargestellt. Eine erregerbezogene Übersicht über entsprechende Therapien und ihre Indikationsstellungen wird aus Tab.2 ersichtlich. Im Folgenden wird näher auf die gezielte 


\begin{tabular}{|c|c|}
\hline \multicolumn{2}{|c|}{$\begin{array}{l}\text { Statistik meldepflichtiger infektiöser Darmkrank- } \\
\text { heiten des RKI für } 2014 \text {. }\end{array}$} \\
\hline Erregerspezies & $\begin{array}{l}\text { Gemeldete Fallzahl } 2014 \\
\text { in Deutschland }\end{array}$ \\
\hline Norovirus-Erkrankung & 74404 \\
\hline Campylobacter-Enteritis & 70446 \\
\hline Rotavirus-Erkrankung & 32224 \\
\hline Salmonellose & 16065 \\
\hline EPEC & 8344 \\
\hline EHEC (außer HUS) & 1623 \\
\hline Giardiasis & 3989 \\
\hline Yersiniose & 2472 \\
\hline Kryptosporidiose & 1713 \\
\hline Shigellose & 550 \\
\hline
\end{tabular}

EHEC: enterohämorrhagische E. coli, EPEC: sonstige enteropathogene E. coli, HUS: hämolytisch-urämisches Syndrom

antimikrobielle Therapie der wichtigsten gastrointestinalen Infektionen eingegangen, mit einer ausführlichen Darstellung der Behandlung von Clostridium-difficileInfektionen (CDI).

\section{- Salmonellose}

Die Inzidenz in Deutschland liegt bei ca. 3/100000. Infektionen durch nicht typhoidale Salmonella-Spezies nehmen in der Regel einen selbstlimitierenden Verlauf und erfordern keine antibiotische Therapie, da hierdurch die Erregerausscheidung verlängert werden kann [14]. Eine gezielte antimikrobielle Therapie ist bei schweren invasiven Verlaufsformen bzw. Sepsis angezeigt. Wegen möglicher, nicht auszuschließender Komplikationen sollte bei Erkrankungen im ersten Lebensjahr, bei hochbetagten Patienten, Personen mit angeborenen oder erworbenen Immundefekten und Patienten mit bekannten Anomalien bzw. prothetischem Ersatz an Herzklappen oder Gefäßen eine antimikrobielle Therapie auch bei einer rein gastroenteritischen Manifestation erwogen werden [15].

Resistenzbestimmung. Aufgrund der auch bei Salmonellen zunehmenden Resistenzentwicklung ist eine kulturelle Resistenzbestimmung des Erregers grund-

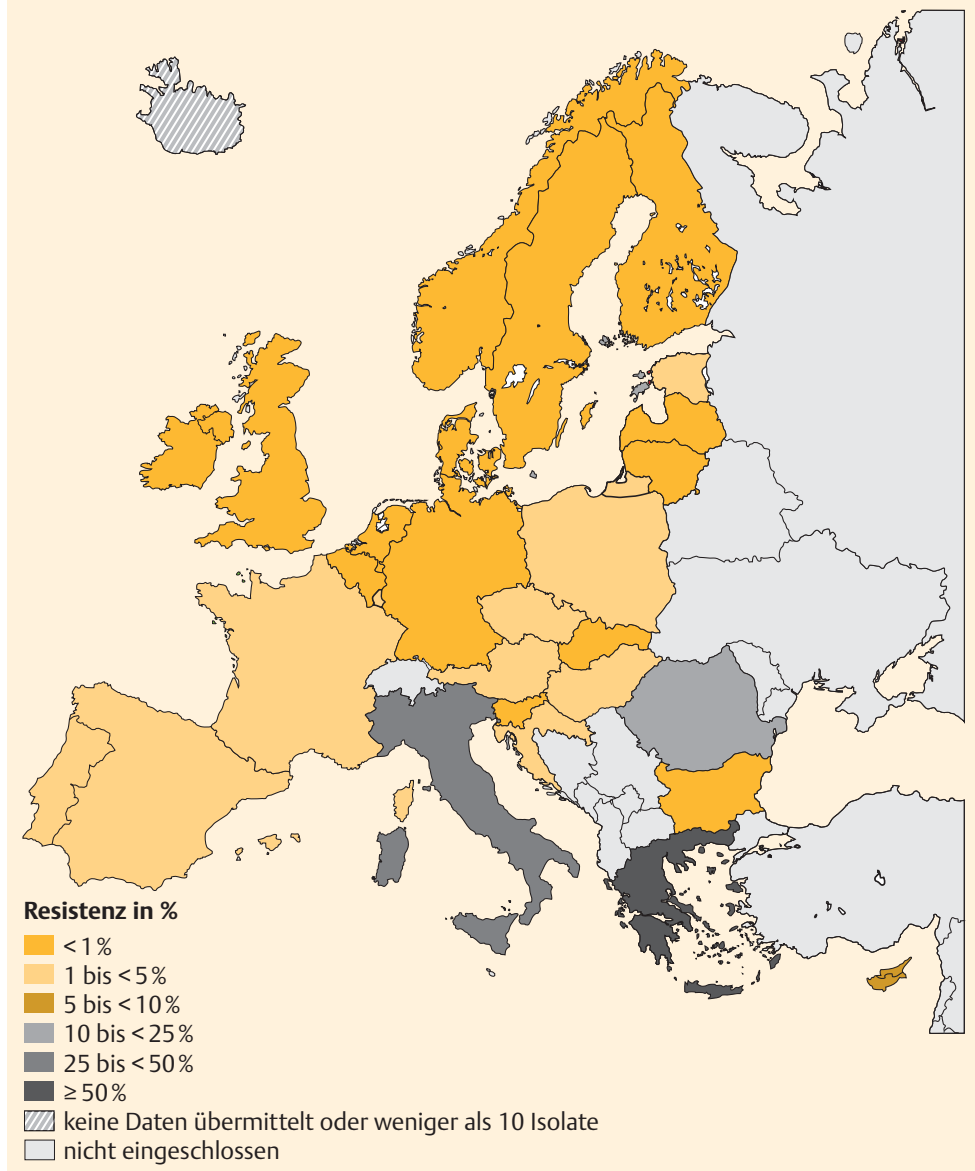

Abb.4 Anteil der Klebsiella-pneumoniae-Isolate mit Resistenz gegenüber Carbapenemen in Europa (ECDC 2015).

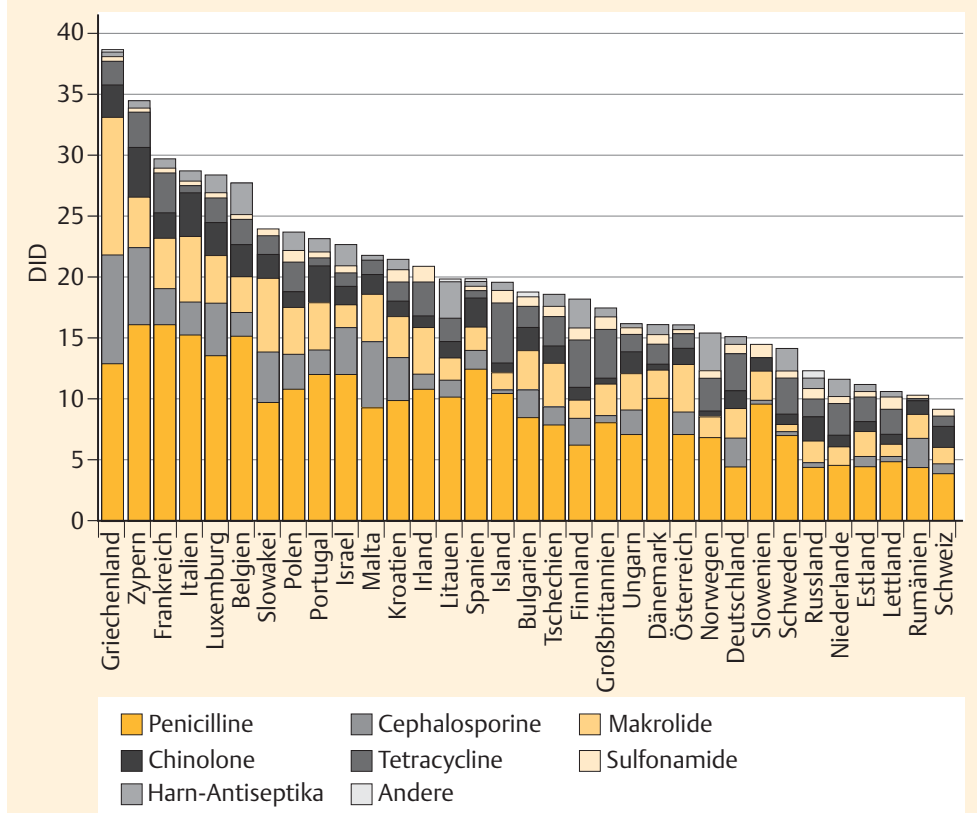

Abb.5 Ambulanter Antibiotikaverbrauch in Europa: tägliche Dosis pro 1000 Einwohner [12]. 
Tabelle 2

Wichtige Erregersteckbriefe und Therapieprinzipien bei infektiöser Diarrhö [1, 38].

\begin{tabular}{|c|c|c|c|c|}
\hline Erreger & Inkubationszeit & Klinische Symptomatik & Krankheitsdauer & Therapie \\
\hline \multicolumn{5}{|l|}{ Viren } \\
\hline Noroviren & ca. 1 Tag & $\begin{array}{l}\text { Erbrechen, Durchfall, Myalgien, } \\
\text { Kopfschmerzen, selten Fieber }\end{array}$ & $1-2$ Tage & symptomatisch \\
\hline Astroviren & & wässrige Durchfälle & 2-3 Tage & symptomatisch \\
\hline Rotaviren & & $\begin{array}{l}\text { wässrige Durchfälle, Erbrechen, } \\
\text { selten Fieber }\end{array}$ & $5-7$ Tage & symptomatisch \\
\hline Adenoviren & & Durchfall, Erbrechen, selten Fieber & bis zu 12 Tage & symptomatisch \\
\hline \multicolumn{5}{|l|}{ Bakterien } \\
\hline Salmonellen & $1-3$ Tage & $\begin{array}{l}\text { Gastroenteritis ( } 75 \%) \text {, Fieber ( } 50 \%) \text {, } \\
\text { Bakteriämie ( } 5-10 \%) \text {, blutige Diarrhö } \\
\text { (gelegentlich); } \\
\text { Komplikation: reaktive Arthritis }\end{array}$ & $1-3$ Wochen & $\begin{array}{l}\text { bei invasiver Erkrankung: } \\
\text { Ciprofloxacin, } \\
\text { Azithromycin }\end{array}$ \\
\hline Shigellen & $1-8$ Tage & $\begin{array}{l}\text { Fieber, starke Bauchschmerzen, } \\
\text { zunächst wässrige, später blutig- } \\
\text { schleimige Diarrhö; } \\
\text { Komplikation: reaktive Arthritis }\end{array}$ & $\begin{array}{l}3 \text { Tage bis } 1 \text { Woche (sel- } \\
\text { ten bis zu } 4 \text { Wochen) }\end{array}$ & $\begin{array}{l}\text { Ciprofloxacin, } \\
\text { Azithromycin }\end{array}$ \\
\hline Yersinien & 1-3 Tage & $\begin{array}{l}\text { Fieber, Bauchschmerzen, Durchfälle; } \\
\text { Komplikation: reaktive Arthritis }\end{array}$ & $1-3$ Wochen & $\begin{array}{l}\text { bei hochakuten Verläufen: } \\
\text { Ciprofloxacin, Doxycyclin }\end{array}$ \\
\hline Campylobacter & $1-4$ Tage & $\begin{array}{l}\text { starke Bauchschmerzen, wässrige } \\
\text { Durchfälle (gelegentlich blutig); } \\
\text { Komplikation: reaktive Arthritis, selten } \\
\text { Guillain-Barré-Syndrom }\end{array}$ & bis 1 Woche & $\begin{array}{l}\text { bei schweren Verläufen: } \\
\text { Azithromycin, } \\
\text { Ciprofloxacin }\end{array}$ \\
\hline $\begin{array}{l}\text { Enterotoxinbildende/ } \\
\text { enteropathogene E. coli } \\
\text { (ETEC, EPEC) }\end{array}$ & $1-2$ Tage & $\begin{array}{l}\text { wässrige Durchfälle, in schweren Fällen } \\
\text { choleraartig (Reiswasser-Stühle) }\end{array}$ & 3-7 Tage & $\begin{array}{l}\text { bei schweren Verläufen: } \\
\text { Ciprofloxacin, } \\
\text { Azithromycin, Rifaximin }\end{array}$ \\
\hline $\begin{array}{l}\text { Enteroinvasive/ } \\
\text { enterohämorrhagische } \\
\text { E. coli (EIEC, EHEC) }\end{array}$ & 1 - 14 Tage & $\begin{array}{l}\text { wässrige oder blutige Diarrhö, Erbre- } \\
\text { chen, Fieber, Bauchschmerzen, hämoly- } \\
\text { tisch-urämisches Syndrom (EHEC) }\end{array}$ & 3-7 Tage & keine Antibiotikatherapie \\
\hline $\begin{array}{l}\text { Bakterielle Toxine von } \\
\text { Staphylococcus aureus }\end{array}$ & Stunden & $\begin{array}{l}\text { Übelkeit, Erbrechen, wässrige } \\
\text { Durchfälle }\end{array}$ & $1-2$ Tage & keine Antibiotikatherapie \\
\hline $\begin{array}{l}\text { Bakterielle Toxine von } \\
\text { Bacillus cereus }\end{array}$ & Stunden & $\begin{array}{l}\text { Übelkeit, Erbrechen, wässrige } \\
\text { Durchfälle }\end{array}$ & $1-2$ Tage & keine Antibiotikatherapie \\
\hline $\begin{array}{l}\text { Bakterielle Toxine von } \\
\text { Clostridium perfringens }\end{array}$ & Stunden & $\begin{array}{l}\text { Übelkeit, Erbrechen, wässrige } \\
\text { Durchfälle, Bauchkrämpfe, } \\
\text { Dehydratation }\end{array}$ & $1-2$ Tage & keine Antibiotikatherapie \\
\hline Vibrio cholerae & $1-3$ Tage & $\begin{array}{l}\text { reiswasserähnliche Stühle und } \\
\text { Erbrechen, massive Exsikkose }\end{array}$ & $\begin{array}{l}\text { individuell sehr } \\
\text { unterschiedlich }\end{array}$ & $\begin{array}{l}\text { Rehydratationsbehandlung } \\
\text { entscheidend, flankiert } \\
\text { durch antimikrobielle The- } \\
\text { rapie mit Ciprofloxacin oder } \\
\text { Azithromycin }\end{array}$ \\
\hline
\end{tabular}




\begin{tabular}{|c|c|c|c|c|}
\hline Tabelle 2 & & & & \\
\hline (Fortsetzung) & & & & \\
\hline Erreger & Inkubationszeit & Klinische Symptomatik & Krankheitsdauer & Therapie \\
\hline Clostridium difficile & unterschiedlich & $\begin{array}{l}\text { meist in direktem zeitlichem Zu- } \\
\text { sammenhang mit vorangegangener } \\
\text { Antibiotikagabe: } \\
\text { 1. wässrige, selbstlimitierende } \\
\text { Durchfälle } \\
\text { 2. pseudomembranöse Kolitis, } \\
\text { potenziell lebensbedrohlich }\end{array}$ & $\begin{array}{l}\text { individuell sehr unter- } \\
\text { schiedlich, u.U. über } \\
\text { Monate }\end{array}$ & $\begin{array}{l}\text { Metronidazol, Vancomycin } \\
\text { (oral), evtl. Fidaxomicin }\end{array}$ \\
\hline Protozoen & & & & \\
\hline Giardia lamblia & 7-10 Tage & Giardiasis / Lambliasis & $\begin{array}{l}\text { unbehandelt bis } \\
\text { mehrere Monate oder } \\
\text { sogar Jahre }\end{array}$ & Metronidazol, Albendazol \\
\hline Entamoeba histolytica & $\begin{array}{l}11-21 \text { Tage, u. } U . \\
\text { deutlich länger }\end{array}$ & $\begin{array}{l}\text { Amöbiasis (Amöbenruhr), blutige } \\
\text { Durchfälle, Erbrechen, Fieber, Bauch- } \\
\text { schmerzen, häufigste extraintestinale } \\
\text { Manifestation: Amöbenleberabszess }\end{array}$ & $\begin{array}{l}\text { unbehandelt bis } \\
\text { mehrere Monate }\end{array}$ & $\begin{array}{l}\text { Metronidazol (bei invasiver } \\
\text { Infektion), Paromomycin } \\
\text { (zur intraluminalen Zysten- } \\
\text { sanierung) }\end{array}$ \\
\hline Cryptosporidium parvum & 3-12 Tage & $\begin{array}{l}\text { wässrige Durchfälle, Bauchschmerzen, } \\
\text { leichtes Fieber }\end{array}$ & $\begin{array}{l}\text { bei Immunkompetenten } \\
\text { selbstlimitierend }\end{array}$ & $\begin{array}{l}\text { bei HIV-Patienten antiretro- } \\
\text { virale Therapie indiziert }\end{array}$ \\
\hline
\end{tabular}

sätzlich anzuraten, insbesondere bei importierten Erkrankungen [1,15]. In Abhängigkeit von den Ergebnissen der Resistenztestung können Fluorchinolone wie Ciprofloxacin oder alternativ Ampicillin, Azithromycin oder Cephalosporine der 3. Generation (z.B. Cefotaxim) mit einer Therapiedauer von 7-10 Tagen, bei Immunsupprimierten 14 Tagen, angewandt werden.

Dauerausscheider. Die genannten Antibiotika eignen sich auch zur Behandlung der bei nicht typhoidalen Salmonellosen nur sehr selten auftretenden Dauerausscheider (Ausscheidedauer länger als $1 \mathrm{Jahr}$ ). In solchen Fällen sollten jedoch zuerst mögliche prädisponierende Faktoren (z.B. Gallensteinleiden) abgeklärt und gezielt behandelt werden. Im Nachgang der 4- bis 6-wöchigen antimikrobiellen Therapie muss der Behandlungserfolg durch mikrobiologische Kontrollen nachgewiesen werden [1].

Salmonellosen sollten nur bei schweren invasiven Verlaufsformen, Sepsis, Risikopatienten sowie Dauerausscheidern nach Resistenzbestimmung antibiotisch behandelt werden.

\section{Shigellose}

In ganz Deutschland werden jährlich ca. 500-1000 Fälle gemeldet. Bei der Shigellose wird aufgrund der hohen Infektiosität eine Antibiotikabehandlung grundsätzlich empfohlen [16]. Die Bakterienausscheidung wird hierdurch reduziert und die Krankheitsdauer verkürzt [17]. Aufgrund der zunehmenden Resistenzentwicklung insbesondere gegenüber Fluorchinolonen ist eine Resistenztestung des Erregers grundsätzlich anzuraten [1,17]. Prinzipiell empfohlen wird die Gabe von Azithromycin 1-mal $500 \mathrm{mg}$ p.o. über 3 Tage oder Ciprofloxacin 2-mal $500 \mathrm{mg}$ p. o. über 3-5 Tage.

Abhängig vom Resistenzprofil kommen alternativ Cephalosporine der 3. Generation (z.B. Cefotaxim) zur Anwendung. Bei Patienten in gutem Allgemeinzustand kann auch eine symptomatische Therapie mit oralem Flüssigkeitsersatz ausreichend sein. Motilitätshemmer sollten zur Behandlung der Shigellose nicht eingesetzt werden.

Die Shigellose sollte wegen der hohen Infektiosität grundsätzlich nach Resistenzbestimmung antibiotisch behandelt werden. Motilitätshemmer sollen nicht verabreicht werden. 


\section{Campylobacter-Infektionen}

Die Inzidenz in Deutschland liegt mit ca. 8/100000 inzwischen deutlich über der von Salmonellosen. Es dominieren Infektionen durch Campylobacter jejuni. In der Regel verläuft die Krankheit selbstlimitierend. Eine symptomatische Therapie mit Volumen- und Elektrolytsubstitution ist in fast allen Fällen ausreichend. Eine antibiotische Therapie ist indiziert bei Patienten mit Zeichen einer invasiven Erkrankung (blutige Diarrhö, Fieber), bei Verdacht auf septische Streuung und schweren klinischen Verläufen (z. B. bei immunsupprimierten Patienten und Persistenz der Symptome für länger als eine Woche) [18]. Mittel der Wahl sind Makrolide (Azithromycin, Clarithromycin) und in zweiter Linie Fluorchinolone (Ciprofloxacin) mit einer Therapiedauer von 3-5 Tagen. Bei Letzteren wird seit ca. 20 Jahren eine zunehmende Resistenzentwicklung beobachtet, die in Ländern wie Thailand mittlerweile bei bis zu >90\% liegt [19]. Leider werden zunehmend auch Makrolid-resistente Campylobacter-Stämme bekannt [20].

Bei den in der Regel selbstlimitierend verlaufenden Campylobacter-Infektionen ist eine Antibiose nur in Ausnahmefällen indiziert (invasive Erkrankung, Sepsis, Immunsuppression).

\section{Pathogene Escherichia-coli-Stämme}

$\mathrm{Zu}$ berücksichtigen sind

- enteropathogene E. coli (EPEC),

- enterotoxinbildende E. coli (ETEC),

- enteroinvasive E. coli (EIEC),

- enterohämorrhagische E. coli (EHEC),

- enteroaggregative E. coli (EAEC) sowie

- diffus adhärente E. coli (DAEC).

EHEC. Enterohämorrhagische E.-coli-Bakterien weisen mehrere Besonderheiten auf, die ihre Virulenz erhöhen: Durch spezielle Hüllproteine (Adhäsine) können sie sich an die Epithelzellen der Darmwand anheften; infolge Phageninfektion mit Gentransfer besitzen sie die Fähigkeit der Produktion von Shiga-Toxin bzw. Vero-Toxin (STEC/VTEC), und schließlich produzieren EHEC-Stämme plasmidkodierte Hämolysine. Auf die 3 häufigsten Serogruppen 0157, 0103 und 026 verteilten sich fast 60\% der Erregernachweise. Der weltweit bislang größte EHEC/STEC-Ausbruch in Deutschland Mitte 2011 wurde hingegen durch einen ShigaToxin produzierenden EAEC-Stamm (STEC 0104:H4) hervorgerufen [21,22].
Antibiotikabehandlung. Die antibiotische Therapie von ETEC-Infektionen als Haupterreger der Reisediarrhö mit Rifaximin, Ciprofloxacin oder Azithromycin bleibt nichtselbstlimitierenden Verläufen vorbehalten. Die Bakterienausscheidung kann hierdurch reduziert und die Krankheitsdauer verkürzt werden [7]. Eine antimikrobielle Therapie bei Patienten mit EHEC bzw. STEC wird nicht empfohlen. Die Gefahr ungünstiger Wirkungen (vor allem bei bereits etablierter Infektion) mit erhöhtem Risiko für ein hämolytisch-urämisches Syndrom (HUS) überwiegt hier deutlich gegenüber der (in der Regel nur bei sehr frühem Einsatz evtl. erzielbaren) raschen Erregerreduktion [1]. Eine derartige Anwendungseinschränkung ist möglicherweise bei neueren Makroliden wie Azithromycin sowie bei Rifampicin/ Rifaximin nicht in gleichem Maße gegeben [22]. Ungünstige Wirkungen in vitro und in vivo wurden bisher auch nicht für Carbapeneme berichtet [21,22]. Eine Therapie mit Azithromycin 1-mal 500 mg p.o. über 3 Tage scheint sich günstig auf die Vermeidung eines längerfristigen EHEC/STEC-Trägerstatus auszuwirken [22].

Eine antibiotische Therapie von Infektionen mit EHEC bzw. STEC wird wegen des dadurch erhöhten Risikos für ein hämolytisch-urämisches Syndrom nicht empfohlen.

\section{Yersiniose}

Die Inzidenz in Deutschland liegt bei ca. 3-5/100000. Infektionen durch Yersinia enterocolitica nehmen in der Regel einen selbstlimitierenden Verlauf und erfordern keine antibiotische Therapie. Nur schwere Verläufe sollten mit Ciprofloxacin 2-mal $500 \mathrm{mg}$ p.o. oder alternativ Doxycyclin 2-mal $100 \mathrm{mg}$ antibiotisch behandelt werden. Bei Vorliegen einer Sepsis kommen Cephalosporine der 3. Generation (z. B. Cefotaxim 3-mal $2 \mathrm{~g}$ i.v.) oder eine Kombinationstherapie aus Doxycyclin 2-mal $100 \mathrm{mg}$ i.v. und Gentamicin $5-7 \mathrm{mg} / \mathrm{kg}$ KG i.v. zum Einsatz $[1,5]$.

\section{Cholera}

Bei der Cholera handelt es sich um eine schwere akute sekretorische Diarrhö verursacht durch Infektion mit Vibrio cholerae, Serogruppe 01 oder 0139. Die Erkrankung ist in mehr als 50 Ländern endemisch und verursacht regelmäßig epidemische Ausbrüche, zuletzt z.B. in Haiti seit dem Jahr 2010 [23]. Therapeutisch entscheidend ist eine suffiziente parenterale Rehydratationstherapie. Nach Deutschland importierte Fälle sind sehr selten. Wegen zunehmender Resistenzen gegenüber Tetracyclin und Doxycyclin werden Ciprofloxacin in einer Dosis von $15 \mathrm{mg} / \mathrm{kg}$ KG i.v. über 3 Tage oder 
Azithromycin in einer Einzeldosis von $20 \mathrm{mg} / \mathrm{kg}$ KG i.v. bevorzugt $[1,23]$.

\section{Clostridium-difficile-Infektionen}

Epidemiologie. Zahl und Schwere der Fälle von C.-difficile-Infektionen (CDI) bzw. C.-difficile-assoziierter Diarrhö (CDAD) steigen weltweit nicht nur im Krankenhaus, sondern auch bei ambulanten Patienten an [24]. Für Deutschland wird eine Inzidenz von mindestens 5-20 Fällen pro 100000 Einwohner und Jahr angenommen [25]. Das höchste Risiko für eine CDI besteht im ersten Monat nach einer Antibiotikatherapie [26].

Therapie. Primärprophylaktisch steht der rationale Einsatz von Antibiotika im Vordergrund. Es ist gut belegt, dass die Reduktion von Antibiotika die Inzidenz von CDI signifikant reduziert [24,26]. In leichten Erkrankungsfällen verschwindet die Symptomatik bereits nach dem Absetzen der verursachenden Antibiotika.

Ist dies nicht möglich oder handelt es sich um mittelschwere bis schwere Fälle (für die es jedoch keine einheitlichen klinischen Definitionen gibt) (Abb.6), stehen in erster Linie Metronidazol (oral oder i.v. verabreicht) und oral gegebenes Vancomycin als Therapie zur Verfügung [24]. Dabei hat sich eine klinische Risikostratifizierung nach Zar et al. [27] bewährt, nach der Patienten mit leichten CDI den gleichen Behandlungserfolg hatten, wenn sie mit 3-mal $500 \mathrm{mg}$ Metronidazol p.o. oder 4-mal $125 \mathrm{mg}$ Vancomycin p. o. behandelt wurden. Bei schweren Verläufen war Vancomycin überlegen $[24,27]$. In der täglichen Praxis bietet sich eine Dosierung von 4-mal $250 \mathrm{mg}$ Vancomycin als Trinklösung aus Ampullen an (Tagestherapiekosten nach Roter Liste 2014 ca. $30 €$ ). Ebenfalls erhältlich in einer Einzeldosis von $250 \mathrm{mg}$ sind Vancomycin-Enterokapseln, wobei jedoch höhere Tagestherapiekosten von ca. $80 €$ (Rote Liste 2014) zu berücksichtigen sind. Kontrollierte Studien zur Behandlung von leichten Fällen mit Metronidazol versus Plazebo existieren nicht, sodass die Frage, inwiefern leichte CDI überhaupt behandelt werden müssen, oder ob nicht ein alleiniges Absetzen des Antibiotikums ausreicht, unter dem Gesichtspunkt der evidenzbasierten Medizin nicht zu beantworten ist. Zu bedenken ist, dass Metronidazol vollständig im oberen Dünndarm resorbiert wird und antimikrobiell wirksame Medikamentenspiegel nur durch Sekretion im entzündeten Kolon erreicht werden. Die i.v. Gabe ist der oralen zumindest gleichwertig [24]. Oral gegebenes Vancomycin hingegen erreicht bereits bei einer Dosierung von 4-mal 125 mg Konzentrationen im Kolon, die

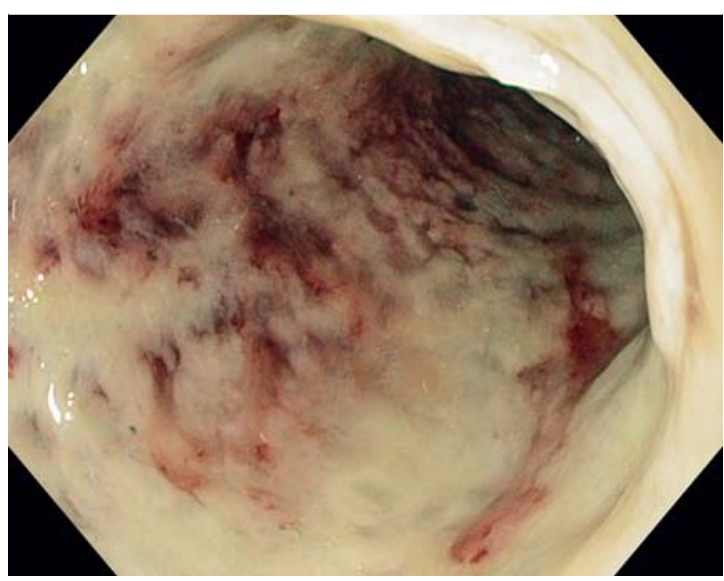

Abb. 6 Endoskopisches Bild einer schweren pseudomembranösen Pankolitis bei einem 71jährigen Patienten.

mehr als 1000-fach über der minimalen Hemmkonzentration (MHK) liegen. Die europäischen Leitlinien zur Behandlung der CDI empfehlen die Gabe von i.v. Metronidazol, wenn eine orale Aufnahme nicht möglich ist, bzw. in Kombination mit Vancomycin bei sehr schweren Fällen [28, 29].

Clostridium-difficile-Infektionen werden je nach Schwere des Krankheitsbildes durch Absetzen der verursachenden Antibiotika, Metronidazol (oral oder i.v.) und Vancomycin (oral) behandelt.

Ansprechen der Therapie. Der Therapieerfolg wird anhand des klinischen Verlaufs beurteilt; Stuhluntersuchungen zur Beurteilung des Therapieerfolgs bei klinischer Besserung sind nicht sinnvoll. In den letzten Jahren gab es zunehmend Berichte über ein Versagen der Standardtherapie mit Metronidazol, sodass vermehrt Vancomycin zum Einsatz kommt [30]. Originaldaten aus dem Universitätsklinikum Leipzig aus den Jahren 2007-2011 zeigen hingegen, dass dieser Trend nicht flächendeckend ist und sich auch nicht zwangsläufig in der mikrobiologischen Austestung widerspiegelt. So scheint die Resistenzlage gegenüber Metronidazol wie Vancomycin in Deutschland weiterhin günstig zu sein [31].

Prophylaxe mit Probiotika. Die prophylaktische Wirksamkeit von Probiotika über Modulation der intestinalen Mikroflora und Funktionsbeeinflussung der Darmmukosa ist Gegenstand von laufender Forschung und Arzneimittelentwicklung. Überzeugende Daten aus größeren kontrollierten Studien lagen hierzu bislang kaum vor [32]. Eine Metaanalyse einer kanadischen Gruppe konnte aber bei Auswertung von 20 klinischen Studien mit mehr als 3800 Patienten, die parallel zu einer Antibiotikaeinnahme verschiedene Probiotika erhalten hatten, gepoolt eine bis zu 66\%ige Risiko- 
Neue Therapieoptionen

Primär- und Rezidivtherapie von C. difficile

Fidaxomicin. Eine insgesamt deutlich geringere Rezidivrate bei primär gleicher Wirksamkeit wie Vancomycin verspricht die Therapie mit dem nicht resorbierbaren makrozyklischen Antibiotikum Fidaxomicin, das 2011 in den USA und Kanada und Ende 2012 auch in Europa zugelassen wurde. Der breite Einsatz dieses Antibiotikums ist sicher auch durch den hohen Preis von ca. $1600 €$ (durchschnittlicher Krankenhausabgabepreis, nach Roter Liste $2200 €)$ für einen 10-tägigen Behandlungszyklus (Dosis: 2-mal $200 \mathrm{mg}$ p. o.) eingeschränkt. Fidaxomicin weist in vitro eine deutlich höhere Aktivität gegen C. difficile als Vancomycin auf und wirkt im Gegensatz zu Vancomycin bakterizid, hinzu kommt ein besserer postantibiotischer Effekt. Minimale intestinale Absorption und geringere Aktivität gegen die physiologische Darmflora als beim Einsatz von Metronidazol oder Vancomycin machen dieses Medikament zu einem vielversprechenden Kandidaten zur Verbesserung und Erweiterung der therapeutischen Optionen. Die Zulassungsstudie von Louie et al. beweist mit 7,8\% vs. $25,5 \%$ erstmalig eine statistisch signifikant geringere Rezidivrate im Vergleich zur Standardtherapie mit Vancomycin, bei vergleichbarem Wirksamkeits- und
Nebenwirkungsprofil [34]. Allerdings konnte dieser Effekt nur für nicht hypervirulente $C$.-difficile-Stämme gezeigt werden. Bei Vorliegen einer Infektion mit dem in Nordamerika dominierenden hypervirulenten Ribotyp 027 war die Rezidivrate unverändert hoch [34]. Vermutlich ist die geringere Rezidivrate unter Fidaxomicin dadurch zu erklären, dass dieses ein engeres antibiotisches Spektrum aufweist, da es z. B. die Bacteroides-Gruppe im Darm nicht supprimiert und die intestinale Flora somit insgesamt weniger beeinflusst wird als bei oraler Vancomycin-Gabe. Auch wenn Fidaxomicin kaum aus dem Darm resorbiert wird, kommt es dennoch zu Nebenwirkungen wie Übelkeit (11\%), Erbrechen (7\%), abdominalen Schmerzen (6\%), gastrointestinalen Blutungen (4\%), Anämie (2\%) oder Neutropenie (2\%) [24, 34].

Mikrobiomtransfer. Eine hochwirksame auch in den europäischen Leitlinien empfohlene Alternative zur medikamentösen Therapie von multiplen CDI-Rezidiven ist der sog. Mikrobiomtransfer (oder fäkale Bakterientherapie, „Stuhltransplantation“), dem Fallanalysen von mehr als 600 Patienten sehr gute Behandlungsergebnisse bei mehrfachem CDI-Rezidiv bescheinigen [24, 29]. Eine langfristige Erfolgsrate von ca. 80 - $90 \%$ wurde durch eine Interimsanalyse einer monozentrischen Studie aus den Niederlanden bestätigt, bei der Spenderstuhl bei 16 Patienten kontrolliert über eine nasoduodenale Sonde verabreicht wurde. Die klinische Erfolgsrate gegenüber der Standardtherapie mit Vancomycin lag bei $81 \%$ vs. $31 \%$ [35].

Rifaximin, Tigecyclin. Zum Einsatz von Rifaximin p.o. und Tigecyclin i.v. liegen bislang leider nur fallbezogene Daten vor [24].

Monoklonale Antikörper. Eine andere, bereits in Phase-III-Studien erfolgreich angewandte therapeutische Option zur Rezidivprophylaxe ist die i.v. Verabreichung monoklonaler Antikörper gegen Toxin A und B. In dieser Studie konnte mit einer einzigen Infusion der Anteil von Rezidiven von $25 \%$ auf $7 \%$ gesenkt werden [36]. Ähnlich wie die Gabe von Fidaxomicin ist dieser Therapieansatz durch hohe Behandlungskosten limitiert [24]. Eine Zulassung in Europa wird für das Jahr 2016 erwartet. reduktion hinsichtlich des Erleidens einer CDI nachweisen [33].

Rezidive. Trotz klinisch erfolgreicher primärer Therapie kommt es bei bis zu 25\% der CDI-Patienten zu einem Rezidiv der Erkrankung. In einem Teil der Fälle handelt es sich möglicherweise um Neuinfektionen, die ohne genaue Erregertypisierung nicht vom echten Rezidiv, d.h. von einer erneuten klinischen Manifestation einer CDI, unterschieden werden können. Mittels spezifischer PCR, die eine Sequenzierung toxinkodierender Gene ermöglicht, lässt sich nachweisen, dass es sich bei 10 - 50\% der Fälle um Neuinfektionen handelt [24,29]. Die Risikofaktoren für ein Rezidiv ähneln denen der Primärinfektion.
Rezidivtherapie. Die derzeitigen Empfehlungen der European Society for Clinical Microbiology and Infectious Diseases (ESCMID) fordern, das erste Rezidiv mit Vancomycin oder alternativ mit Fidaxomicin zu behandeln (s. „Neue Therapieoptionen - Primär- und Rezidivtherapie von C. difficile“) [29]. Bei multiplen Rückfällen kann z.B. ein Vancomycin-Reduktionsschema durchgeführt werden. Ein mögliches Schema, das bereits erfolgreich von den Autoren angewandt wurde, ist: 4-mal $125 \mathrm{mg}$ Vancomycin p.o. pro Tag für 10-14 Tage, gefolgt von 2-mal $125 \mathrm{mg}$ Vancomycin p.o. pro Tag für 7 Tage, gefolgt von 1-mal $125 \mathrm{mg}$ Vancomycin p.o. pro Tag für 7 Tage, gefolgt von 1-mal $125 \mathrm{mg}$ Vancomycin p.o. alle 3 Tage für $2-8$ Wochen $[24,31]$. 


\section{Chronische infektiöse Diarrhöen}

Die Differenzialdiagnosen bei Vorliegen einer chronischen Diarrhö sind vielfältig und umfassen neben den chronisch entzündlichen Darmerkrankungen (CED) Colitis ulcerosa und Morbus Crohn auch gastrointestinale Tumoren, exokrine Pankreasinsuffizienz, vaskuläre und funktionelle Enteropathien, kollagene und lymphozytäre Kolitis, Sprue, Laktose- bzw. Fruktoseintoleranz, autonome Neuropathie bei Diabetes mellitus, Schilddrüsenfunktionsstörungen, intestinale Resorptionsstörungen (z. B. Kurzdarmsyndrom) und medikamentös induzierte oder bestrahlungsassoziierte Enteropathien (z.B. radiogene Kolitis) [37].

Unter den insgesamt seltenen erregerbedingten Krankheitsbildern steht die Lambliasis/Giardiasis im Vordergrund, gefolgt von Amöbenkolitis, Kryptosporidiose bzw. Mikrosporidiose bei immunsupprimierten Patienten und dem sehr selten auftretenden Morbus Whipple.

\section{- Lambliasis/Giardiasis}

Die Inzidenz einer Infektion mit Giardia lamblia (Abb. 7) in Deutschland liegt bei ca. 5/100000. Die Therapie erfolgt in erster Linie mit Metronidazol oder anderen Nitroimidazolpräparaten wie Tinidazol oder auch Secnidazol (vor allem in Südamerika). Letztere sind jedoch in Deutschland nicht im Handel. Die empfohlene Dosierung bei Erwachsenen liegt für Metronidazol bei 3-mal 500 mg p. o. für 5-10 Tage [38]. Im Gegensatz zur Amöbiasis scheint es nach neueren Beobachtungen bei den humanpathogenen Lamblien neuerdings auch Resistenzen gegen Metronidazol zu geben, vor allem auf dem indischen Subkontinent [39]. Eine systematische Resistenztestung wie bei Bakterien wird es auf längere Sicht für die Routine jedoch nicht geben. Sie setzt u.a. die kulturelle Anzüchtung voraus, die nur in wenigen tropenmedizinischen Zentren wie Hamburg, Würzburg und Berlin im Rahmen wissenschaftlicher Fragestellungen durchgeführt wird [38].

Therapieversagen. Bei einem Therapieversagen wird daher empfohlen, eine Behandlung mit medikamentösen Alternativen wie Albendazol, Paromomycin oder Nitazoxanid durchzuführen [40]. Letzteres ist in Deutschland nicht im Handel und scheint nach bisherigen Erfahrungen keine bessere Wirksamkeit im Vergleich mit den vorgenannten Medikamenten zu besitzen. Die empfohlenen Dosierungen bei Erwachsenen liegen für Albendazol bei 1-mal $400 \mathrm{mg}$ p.o. über $5-10$ Tage, für Paromomycin bei täglich $25-30 \mathrm{mg} / \mathrm{kg}$ KG p.o. verteilt auf 3 Tagesdosen über 7-10 Tage und

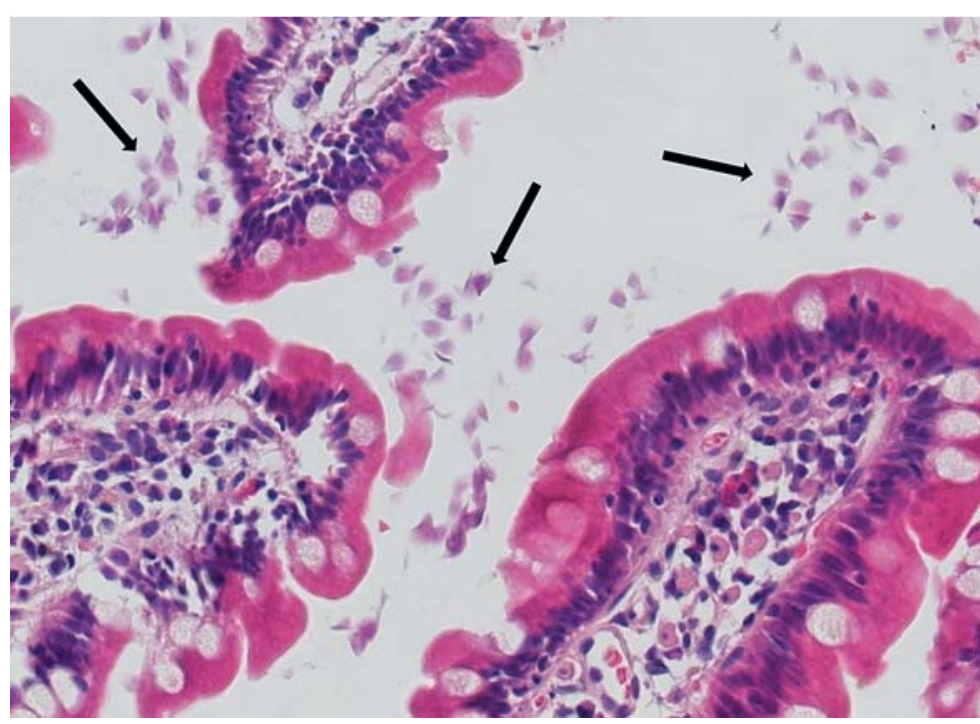

Abb. 7 Dünndarmhistologie mit Nachweis von Giardia lamblia (HE-Färbung, freundlicherweise zur Verfügung gestellt von Prof. Dr. Christian Wittekind, Leipzig).

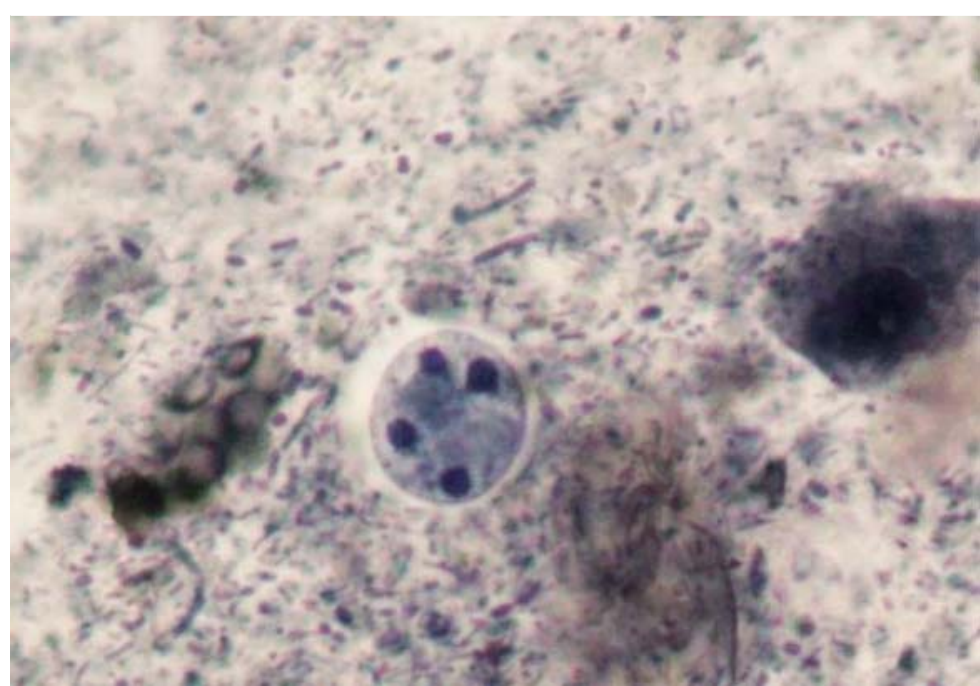

Abb. 8 Mikroskopisches Bild einer vierkernigen Zyste von Entamoeba histolytica (Eisenhämatoxylin-Färbung nach Heidenhain (freundlicherweise zur Verfügung gestellt von Prof. Dr. Stefan Schubert, Leipzig).

für Nitazoxanid bei 2-mal $500 \mathrm{mg}$ p. o. über 3 Tage [38, 40].

\footnotetext{
Die Therapie der Lambliasis erfolgt in erster Linie mit Metronidazol, bei Therapieversagen (Resistenz) vor allem mit Albendazol oder Paromomycin.
}

\section{Amöbenkolitis}

Bis auf wenige Ausnahmen ist die Therapie einer intestinalen Infektion mit Entamoeba histolytica (Abb.8) medikamentös-konservativ. Metronidazol als Mittel der Wahl wirkt sehr gut gegen die gewebsinvasiven Formen, jedoch nicht ausreichend bei reiner Darm- 
lumeninfektion [41,42]. Daher werden anschließend zur Sanierung einer möglicherweise zurückbleibenden Darmlumeninfektion nicht resorbierbare sog. Kontaktamöbizide wie Diloxanid oder Paromomycin verabreicht [38]. Resistenzen sind bei allen genannten Mitteln bisher nicht bekannt geworden. Bei schweren dysenterischen Erkrankungen sind in Einzelfällen zusätzlich Antibiotika gegen bakterielle Superinfektionen angezeigt.

Bei den weitaus häufigeren reinen Darmlumeninfektionen wäre eine Behandlung mit Paromomycin oder einem anderen darmlumenwirksamen Medikament ausreichend. Da sich aber nicht immer sicher eine bereits eingetretene Gewebsinvasion durch einzelne Amöben ausschließen lässt, wird oftmals eine zusätzliche Metronidazol-Behandlung durchgeführt. Je nach klinischer Ausprägung (milde Durchfälle bis akutdysenterisches Stadium, Kolonamöbom) wird bei Erwachsenen Metronidazol in einer Dosis von täglich 3-mal $500 \mathrm{mg}$ p.o. über 7-10 Tage bis täglich 3-mal $10 \mathrm{mg} / \mathrm{kg}$ KG i.v. über 10 Tage verordnet, anschließend Paromomycin täglich $25-30 \mathrm{mg} / \mathrm{kg} \mathrm{KG}$ p.o. verteilt auf 3 Tagesdosen über $7-10$ Tage $[38,41,42]$.

Die Amöbenkolitis wird sequenziell mit Metronidazol und im Anschluss mit Paromomycin (wegen evtl. persistierender Amöben im Darmtrakt) therapiert.

\section{Morbus Whipple}

Die Inzidenz dieser sehr seltenen Erkrankung infolge Infektion mit Tropheryma whipplei in Deutschland liegt bei unter 1/100000. Bislang galt als Behandlung der ersten Wahl die Gabe eines liquorgängigen Breitspektrumantibiotikums (z. B. Cefotaxim 3-mal 2g i.v.) über 2 Wochen mit daran anschließender Erhaltungstherapie bzw. Rezidivprophylaxe mit Cotrimoxazol 2mal $960 \mathrm{mg}$ p.o. oder einem Tetracyclin (z. B. Doxycyclin 2-mal $100 \mathrm{mg}$ p.o.) über mindestens 12 Monate $[38,43]$. Auch kürzere Therapieschemata mit Aminopenicillinen (z.B. Amoxicillin 3-mal $1 \mathrm{~g}$ p.o.) oder Tetracyclinen über nur 8 Wochen scheinen nach kleineren Fallserien ausreichend wirksam zu sein [43].

Therapieerfolg. Der Therapieerfolg sollte durch Kontrollendoskopien überwacht werden. Jedoch gibt es immer wieder Patienten mit Morbus Whipple, die auf die antibiotische Therapie ungenügend ansprechen und wiederkehrende Krankheitsschübe erleiden [38]. Diese Patienten weisen häufig einen ausgeprägten Befall des ZNS auf und zeigen einen ungünstigen klinischen Verlauf, der nicht selten letal endet.

\section{Spezielle Erkrankungen bei Immunsuppression}

Zu berücksichtigen sind neben der ZytomegalievirusInfektion des Kolons vor allem die Folgen einer HIVInfektion.

\section{HIV-Enteropathie und HIV-assoziierte Infektionen des GI-Trakts}

Opportunistische Infektionen. Humane Immundefizienz-Viren (HIV) befallen in der Darmschleimhaut CD4-positive Lymphozyten (T-Helferzellen) und Makrophagen. Die dadurch hervorgerufenen Störungen im spezialisierten Immunsystem des GI-Trakts können zur Ausbreitung sonst eher harmloser, opportunistischer Durchfallerreger wie z.B. den Protozoen Cryptosporidium parvum oder Mikrosporidien wie Enterocytozoon intestinalis führen $[38,44,45]$. Eine Infektion mit diesen Erregern kann im Stadium AIDS unbehandelt tödlich verlaufen.

HIV-Enteropathie. Neben opportunistischen Infektionen kann es im Rahmen der HIV-Infektion schon sehr früh zu Veränderungen im Aufbau der Dünndarmschleimhaut kommen, die für die Resorption von Nahrungsbestandteilen verantwortlich ist. Klinisch zeigt sich eine meist ausgeprägte Durchfallsymptomatik. Mikroskopisch imponiert eine funktionell bedeutsame Zottenatrophie. Diese Beobachtungen führten bereits Ende der 1980er Jahre zur Prägung des Begriffs der HIV-Enteropathie und erklären u.a. den schlechten Ernährungszustand von HIV-Patienten mit fortgeschrittenem Immundefekt $[44,45]$. Die Diagnose einer HIV-Enteropathie wird in erster Linie klinisch gestellt.

Hochaktive antiretrovirale Therapie. Seit Mitte der 1990er Jahre konnte durch die Kombination mehrerer gegen HIV gerichteter Medikamente mit unterschiedlichen Wirkmechanismen im Rahmen der sog. hochaktiven antiretroviralen Therapie (HAART oder ART) ein entscheidender Fortschritt in der Behandlung der HIV-Infektion erzielt werden. Die Therapie führt durch anhaltende Suppression der Vermehrung von HIV auch im GI-Trakt zu einer Regeneration des intestinalen Immunsystems und zu einer weitgehenden Wiederherstellung des Aufbaus sowie der Funktion der Dünndarmschleimhaut $[38,44,45]$. Die medikamentös erzielte Immunrekonstitution bewirkt ferner eine Ausheilung von zuvor nicht therapierbaren chronischen Darminfektionen, z. B. der Kryptosporidiose [46]. In diesem Zusammenhang kann die ART als primäre Therapie angesehen werden. Allerdings können eine Reihe von im Rahmen der ART eingesetzten Substanzen, ins- 
besondere Medikamente aus der Gruppe der Proteaseinhibitoren wie Lopinavir, als typische Nebenwirkung selbst eine milde chronische Diarrhö hervorrufen [38].

HAART führt bei HIV-Patienten zu einer Immunrekonstitution, die u.a. auch die Ausheilung chronischer opportunistischer Darminfektionen ermöglicht.

Häufigste Erreger einer Diarrhö. Kryptosporidien und Isospora belli sind die häufigsten intestinalen Durchfallerreger bei HIV-Patienten [46]. Klinisch stehen chronischer Durchfall, Bauchschmerzen und Gewichtsverlust im Vordergrund. Der Nachweis von Kryptosporidien oder Mikrosporidien im Stuhl setzt den Einsatz von Spezialfärbungen oder PCR-Verfahren voraus.

\section{Kryptosporidiose}

Cryptosporidium hominis und C. parvum sind die häufigsten Erreger der humanen Kryptosporidiose, einer Erkrankung, die bis zu 7\% der akuten Diarrhöen verursacht $[38,47]$. Klinisch imponiert die Kryptosporidiose bei immunkompetenten Patienten als selbstlimitierender, mit Übelkeit und Bauchschmerzen einhergehender wässriger Durchfall. Vor allem Kinder und ältere Menschen sind betroffen. Bei immunsupprimierten Patienten allerdings kann eine Cryptosporidium-Infektion lebensbedrohlich oder chronisch verlaufen [38]. Wie bei der Lambliasis scheint bereits die Aufnahme von 10 Oozysten für eine Infektion ausreichend zu sein [48]. Die Infektion erfolgt in der Regel über verunreinigtes Trinkwasser [38].

Therapie. Die erregerspezifische Therapie der Kryptosporidiose ist schwierig. In einer Metaanalyse von 2007 konnten die Autoren zwar 7 Studien mit insgesamt 169 immunkompromittierten Patienten identifizieren, mussten aber eine fehlende Evidenz für die medikamentöse Behandlung unter diesen Bedingungen feststellen [47]. Bei immunkompetenten Patienten kann Nitazoxanid erfolgreich eingesetzt werden. Die Autoren schlussfolgern, dass aufgrund des fatalen Ausgangs der Erkrankung bei immunkompromittierten Patienten trotz fehlender Evidenz Nitazoxanid ebenfalls eingesetzt werden sollte. Eine Kombination von Paromomycin und Azithromycin erscheint ebenfalls möglich, ohne dass dafür eine hinreichende Evidenz gegeben wäre [38].

Eine Cryptosporidium-Infektion kann bei immunsupprimierten Patienten lebensbedrohlich sein. Trotz fehlender Evidenz bei Immunsuppression wird der Einsatz von Nitazoxanid angeraten.

\section{Mikrosporidiose}

Als Mikrosporidiose werden opportunistische Infektionen mit den intrazellulären Erregern Enterocytozoon bieneusi (bei HIV-Infizierten $>80 \%$ der Fälle) und seltener Encephalitozoon intestinalis bezeichnet. Sie treten in erster Linie bei Vorliegen eines fortgeschrittenen zellulären Immundefekts mit CD4-Zellzahl $<50 / \mu \mathrm{l}$ auf. Die Übertragung erfolgt über verunreinigtes Wasser und Lebensmittel.

Therapie. E. bieneusi kann prinzipiell mit dem in Deutschland nicht zugelassenen Fumagillin behandelt werden $[38,48]$. Die Therapie ist relativ toxisch und erfordert eine Dosierung von täglich 3-mal $20 \mathrm{mg}$ p.o. über 14 Tage. Infektionen durch E. intestinalis sprechen auf die Gabe von Albendazol in einer Dosierung von 2-mal 400 mg über mindestens 3 Wochen an.

\section{Isosporiasis und Cyclosporiasis}

Isospora belli und Cyclospora cayetanensis können bei immunkompetenten Patienten flüchtige Durchfallerkrankungen ohne spezielle Therapiebedürftigkeit hervorrufen. Die Infektion erfolgt über kontaminiertes Wasser oder Lebensmittel bzw. als Schmierinfektion. Bei Vorliegen einer Immunsuppression, insbesondere einer HIV-Infektion mit fortgeschrittenem zellulärem Immundefekt, können I. belli und C. cayetanensis selten zu einem Kryptosporidiose-ähnlichen Krankheitsbild mit chronischer Diarrhö und Malabsorption führen $[38,48]$. Genaue Zahlen zur Inzidenz sind nicht verfügbar. Diagnostisch müssen spezielle Färbungen sowie Stuhlanreicherungsverfahren oder Dünndarmbiopsien eingesetzt werden. Die Isosporiasis kann mit einer milden Bluteosinophilie einhergehen [38].

Therapie. Therapeutisch wirksam ist bei der Isosporiasis wie auch der Cyclosporiasis Cotrimoxazol in einer Dosierung von 4-mal $960 \mathrm{mg}$ über mindestens 7-10 Tage [48]. Vermutlich profitieren die Patienten aufgrund einer Vermeidung von Rezidiven von einer längeren Therapiedauer. Eine längerfristige, niedriger dosierte Suppressionsbehandlung bzw. Rezidivprophylaxe, z.B. mit $960 \mathrm{mg}$ Cotrimoxazol 3-mal pro Woche, kann bei Vorliegen eines fortgeschrittenen zellulären Immundefekts erforderlich sein [38].

Die Mikrosporidiose kann mit Fumagillin behandelt werden. Bei immunkompromittierten Patienten werden die Isosporiasis und die Cyclosporiasis mit Cotrimoxazol therapiert. 


\section{Andere Infektionen des GI-Trakts und seiner „Anhangsorgane“}

Neben bakteriellen Infektionen des Bauchraums (Peritonitis) sowie der Darmwand (z. B. Divertikulitis) besitzen die Helicobacter-pylori-Infektion des Magens, Infektionen der Leber (z. B. Leberabszesse) und der Gallenwege (Cholezystitis, Cholangitis) sowie sekundäre Infektionen des Pankreas einen großen Stellenwert.

\section{Spontane bakterielle Peritonitis}

Pathophysiologie und Klinik. Die spontane bakterielle Peritonitis (SBP) ist fast ausschließlich eine Erkrankung von Patienten mit Leberzirrhose und Aszites (s. „Prophylaxe der SBP-Antibiotika zur Primär- und Sekundärprophylaxe“). „Spontan“ bedeutet im engeren Sinne, dass kein Infektionsfokus eruierbar ist, sondern eine Translokation von intestinalen Erregern über das Blutund Lymphsystem in den Aszites vorliegt [49]. Klinisch stehen Abdominalschmerzen, Zunahme des Aszites, Fieber, Entzündungszeichen, aber auch das Neuauftreten oder die Verschlechterung einer hepatischen Enzephalopathie im Vordergrund $[49,50]$.

Diagnose und Therapie. Die Diagnosestellung der SBP erfolgt mittels Parazentese mit Nachweis von Erregern - typischerweise monobakteriell mit Dominanz von Enterobakterien wie E. coli oder K. pneumoniae im Aszites bzw. indirekt durch eine erhöhte Konzentration von polymorphkernigen Granulozyten

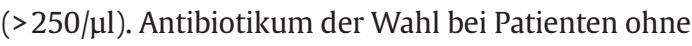
spezielle Risikofaktoren oder Antibiotikavorbehand-

\section{Prophylaxe der SBP}

\section{Antibiotika zur Primär- und Sekundärprophylaxe}

Primärprophylaktisch wird die

Gabe von Cephalosporinen der

3. Generation für alle Patienten mit Leberzirrhose und gastrointestinaler Blutung für 5- 7 Tage empfohlen [50-52]. Norfloxacin (1-mal $400 \mathrm{mg}$ p. o.) wird auch zur Primärprophylaxe der SBP bei Patienten mit fortgeschrittener Zirrhose eingesetzt und konnte in Studien das Risiko für eine SBP und für das Auftreten eines hepatorenalen Syndroms (HRS) senken sowie das 1-Jahres-Überleben der Patienten signifikant verbessern [53]. Die Pri- märprophylaxe der SBP bei Patienten mit Leberzirrhose und verminderter Proteinkonzentration im Aszites (<15 g/l) sowie die Sekundärprophylaxe nach stattgehabter SBP mit Norfloxacin (1-mal 400 mg p. o. täglich) vermochten die SBPRezidivrate signifikant zu senken $[53,54]$. Allerdings ist dieser Ansatz nicht unumstritten, da eine Fluorchinolon-Prophylaxe das Risiko für Infektionen durch multiresistente Erreger, die mit einer höheren Mortalitätsrate assoziiert sind, erhöht [55]. lung sind hierbei Cephalosporine der 3. Generation (z.B. Cefotaxim 3-mal $2 \mathrm{~g}$ i.v.). Alternativ ist bei Patienten ohne hepatische Enzephalopathie und stabiler Nierenfunktion auch eine orale Gabe von Fluorchinolonen wie Norfloxacin (2-mal $400 \mathrm{mg}$ p.o.) möglich [50-52]. Bei Patienten mit Antibiotikavortherapie ist eine kalkulierte antimikrobielle Therapie unter besonderer Berücksichtigung der lokalen Resistenzsituation erforderlich [50].

\section{Sekundäre Peritonitis}

Pathophysiologie und Klinik. Sekundäre bakterielle Peritonitiden sind definiert als Infektionen der Bauchhöhle mit einem chirurgisch sanierbaren Fokus, meist infolge einer Hohlorganperforation im Bereich des GITrakts [56]. Häufige Auslöser sind Ulcera duodeni und ventriculi, Appendizitis oder Divertikulitis. Im Gegensatz zur SBP imponiert die sekundäre Peritonitis als polymikrobielle Infektion des Bauchraums mit ausgeprägten systemischen Entzündungszeichen. Die Mortalitätsrate der sekundären Peritonitis ist trotz adäquater chirurgischer und antibiotischer Therapie hoch und wird in einzelnen Studien mit bis zu 50\% angegeben [57].

Therapie. Die Therapie beruht auf der chirurgischen Sanierung des Fokus und der Gabe von BreitspektrumAntibiotika, die auch Anaerobier erfassen müssen (z.B. 3-mal $2 \mathrm{~g}$ Cefotaxim i.v. plus 3-mal $500 \mathrm{mg}$ Metronidazol i.v., 3-bis 4-mal 4,5g Piperacillin/Tazobactam i.v. oder 4-mal $500 \mathrm{mg}$ Imipenem/Cilastatin i.v.) Eine fehlende Fokussanierung (,failure of source control“) geht in Studien mit einer Verdoppelung der Mortalitätsrate einher [58].

Neben der Gabe von Breitspektrum-Antibiotika ist bei sekundärer Peritonitis die chirurgische Fokussanierung unabdingbar.

\section{Divertikulitis}

Die Prävalenz von Kolondivertikeln liegt bei $33-50 \%$ in der Altersgruppe der über 50-Jährigen. Zu ca. $95 \%$ ist davon das Sigma betroffen (sog. „Hochdruckzone“), seltener weiter proximal liegende Kolonabschnitte [59]. Komplikationen treten nur in 15-20\% der Fälle auf, wobei die bakteriell bedingte Divertikulitis überwiegt. Durch geeignete Bildgebung (abdominelle CT, evtl. spezialisierter Ultraschall) müssen klassische Komplikationen wie Abszessbildung, Perforation oder Fistelbildung erfasst bzw. ausgeschlossen werden. 
Therapie. Bei unkomplizierter Divertikulitis ist eine Antibiotikatherapie nach neuerer Studienlage nicht notwendig [60], sondern eine Nahrungskarenz bis zum Eintritt einer symptomatischen Besserung ausreichend. Bei Vorhandensein einer Peridivertikulitis und bei Nachweis eines phlegmonösen Entzündungsmusters kommen Antibiotika zum Einsatz, deren Wirkspektrum Enterobakterien und Anaerobier umfasst (z.B. 3-mal 3 g Ampicillin/Sulbactam i.v. oder 3-mal $2 \mathrm{~g}$ Cefotaxim i.v. plus 3-mal $500 \mathrm{mg}$ Metronidazol i.v., alternativ 2-mal $400 \mathrm{mg}$ Ciprofloxacin i.v. plus 3-mal 500 mg Metronidazol i.v., jeweils über 7-10 Tage). Resistente Enterobakterien und Enterokokken können Grund für ein Wirkversagen der empirischen Antibiotikatherapie sein, sodass die Eskalationsstufen Piperacillin/Tazobactam in einer Dosierung von 3-bis 4-mal $4,5 \mathrm{~g}$ i.v. bzw. Imipenem/Cilastatin in einer Dosierung von 4-mal $500 \mathrm{mg}$. i.v. umfassen. Eine geeignete Erregerdiagnostik mittels Blutkultur oder ggf. Abszesspunktat ist unbedingt anzustreben.

Komplikationen wie (gedeckte) Perforation und Abszessbildung müssen operativ versorgt werden, sodass die Antibiotikatherapie hier nur adjuvanten Charakter besitzt.

Im Gegensatz zur unkomplizierten Divertikulitis erfordern eine Peridivertikulitis und phlegmonöse Entzündungen eine antibiotische Therapie gegen Enterobakterien und Anaerobier.

\section{Cholezystitis}

Cholezystektomie. Die akute Cholezystitis ist definiert als eine Entzündung der Gallenblase, die typischerweise durch Gallensteinleiden verursacht wird. Die Behandlung der Wahl besteht in der frühzeitigen (max. $72 \mathrm{~h}$ nach Auftreten der Symptome), vorzugsweise laparoskopischen Cholezystektomie [61,62]. Somit ist die frühe operative Intervention und Sanierung mit einer verringerten Mortalitäts- und Komplikationsrate assoziiert [62].

Gabe von Antibiotika. Die primär operierte akute Cholezystitis sollte allenfalls kurzzeitig adjuvant antibiotisch behandelt werden. In den „Tokyo Guidelines“ werden dazu schwere Verlaufsformen (Grad 3) mit Organversagen (neurologische, hämatologische, hepatische, respiratorische, renale oder kardiale Komplikationen) von moderaten Verlaufsformen (Grad 2) mit Zeichen einer prolongierten Entzündungsreaktion (Krankheitsdauer $>72$ h, Leukozytose $>18000 / \mu l$, Persistenz peritonitischer Beschwerden) und von beni- gnen Verläufen (Grad 1) unterschieden, für die keine der für Grad 2 und 3 aufgestellten Kriterien gelten [63].

Die Gabe von Antibiotika (z. B. Amoxicillin/Clavulansäure) nach erfolgter Cholezystektomie vermag die Rate an postoperativen Infektionen (15\% vs. 17\%) nicht zu verringern [64]. Diese Studienergebnisse sollten aber nicht generell zur Anwendung kommen, da größere Untersuchungen zu dieser Frage noch ausstehen und die kompetente, individualisierte klinische Einschätzung nicht ersetzen können. Auch bei leichtgradiger Cholezystitis, die im Intervall operiert wurde, führte die Gabe von Antibiotika nicht zu einer verkürzten Krankenhausverweildauer oder einer geringeren Rate an stationären Wiederaufnahmen [65]. Es ist davon auszugehen, dass es sich bei milde verlaufenden Cholezystitiden primär um inflammatorische Krankheitsbilder handelt, bei denen es allenfalls sekundär zu therapiebedürftigen bakteriellen Infektionen kommen kann.

Eine antibiotische Behandlung nach Cholezystektomie aufgrund akuter Cholezystitis sollte allenfalls kurzzeitig adjuvant bzw. bei schweren Verlaufsformen zum Einsatz kommen.

\section{Cholangitis}

Akute Cholangitiden entstehen in der Regel durch bakterielle Infektion obstruierter Gallenwege. Der Galleabfluss kann dabei durch Gallengangsteine, benigne oder maligne Stenosen bzw. Strikturen sowie in tropischen Regionen durch bestimmte Parasiten (z. B. Ascaris lumbricoides, Fasciola hepatica, Opisthorchis viverrini) behindert werden [66].

Klinik und Erregerspektrum. Die klassischen klinischen Zeichen der akuten Cholangitis werden als CharcotTrias beschrieben und bestehen aus Fieber, rechtsseitigem Oberbauchschmerz und Ikterus. Bei der in der angloamerikanischen Literatur beschriebenen Reynolds-Pentade handelt es sich um schwere Verlaufsformen der Erkrankung mit zusätzlich Hypotension und Verwirrtheitszuständen. Gramnegative Enterobakterien wie E. coli, Klebsiella spp. oder Enterobacter spp. werden am häufigsten bei ambulant erworbenen Cholangitiden isoliert, gefolgt von Enterokokken [56]. Bei Patienten mit nosokomial erworbenen Cholangitiden unterscheidet sich das Erregerspektrum deutlich. So können hier Staphylococcus aureus, Vancomycin-resistente Enterokokken, multiresistente Enterobakterien 

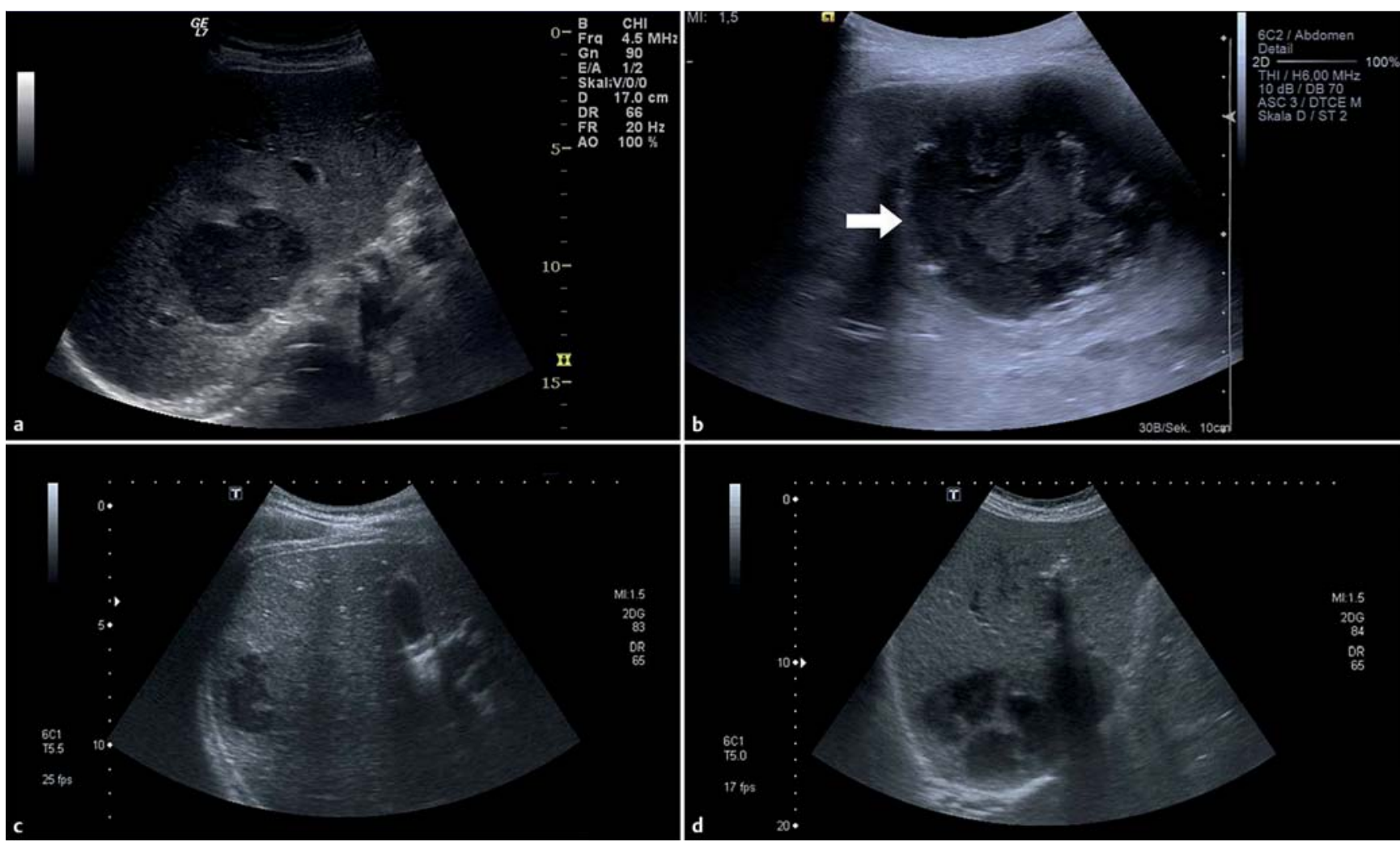

Abb.9 Ultraschall-Morphologie von Leberabszessen (B-Modus): Pyogene Leberabszesse zeigen häufig ein inhomogenes echoarmes Muster (a) mit einer verdickten, ödematösen Wand (b). Unscharfe, unregelmäßige Grenzen (c) und Septen (d) sind ebenfalls zu beachten. Die Ultraschall-Morphologie vermag nicht sicher zwischen pyogenen Abszessen (a, b, d) und einem Amöbenleberabszess (c) zu diskriminieren (freundlicherweise zur Verfügung gestellt von Dr. Thomas Karlas, Leipzig).

und Pseudomonaden gehäuft nachgewiesen werden. Bei vielen Patienten verläuft die Infektion dabei polymikrobiell unter Beteiligung von Anaerobiern $[56,67]$.

Therapie. Die Therapie der akuten Cholangitis umfasst primär die interventionelle Behebung der Cholestase in Kombination mit adjuvanter systemischer Antibiotikatherapie. Letztere sollte frühzeitig begonnen werden [67]. Klinisch milde Verläufen werden meist monobakteriell hervorgerufen. Eine kurzfristige Antibiotikagabe (max. 2-3 Tage) mit gut gallegängigen BetalaktamAntibiotika (Mezlocillin, ggf. Piperacillin/Tazobactam) ist ausreichend $[56,66,67]$. Prolongierte und schwere Verläufe sind häufiger polymikrobiell bedingt und sollten über 5-7 Tage behandelt werden, bei persistierender Cholestase auch länger. Für die Behandlung stehen i.v. appliziertes Piperacillin/Tazobactam, Carbapeneme (vorzugsweise Imipenem/Cilastatin) oder bei bakterieller Multiresistenz ggf. auch Tigecyclin zur Verfügung. Ein Erregernachweis aus Galleaspirat oder Blutkultur mit mikrobiologischer Resistenztestung ist zwingend anzustreben.
Bei akuter Cholangitis wird die interventionelle Behebung der Cholestase kombiniert mit einer adjuvanten systemischen Antibiotikatherapie, die früh begonnen werden soll.

\section{Leberabszess}

Leberabszesse sind eine seltene, jedoch potenziell lebensbedrohliche Erkrankung mit relevanter Morbidität und Mortalität.

Erregerspektrum und Diagnostik. Pyogene Leberabszesse (Abb.9) sind mit über 80\% die häufigste Entität in industrialisierten Gebieten, während Entamoeba histolytica in ca. $10 \%$ der Fälle als Erreger identifiziert werden kann [68]. In tropischen Gebieten ist die Inzidenz der Amöbenleberabszesse deutlich höher. Hochvirulente Klebsiella-pneumoniae-Stämme haben sich in Ost- und Südostasien zur Hauptursache pyogener Leberabszesse entwickelt [68]. Die zunehmende Verbreitung von K. pneumoniae auch in den USA, Australien und europäischen Ländern stellt eine Herausforderung für Behandlung und Prävention von Sekundärkomplikationen dar. Bildgebung (Ultraschall, CT) sowie 
gezielte mikrobiologische Untersuchung (Abszesspunktat, Blutkulturen) sind die wichtigsten diagnostischen Verfahren bei klinischem Verdacht auf einen Leberabszess.

Therapie. Das multimodale Management beinhaltet die Therapie mit einem Breitspektrum-Antibiotikum (z.B. 3-mal $2 \mathrm{~g}$ Cefotaxim i.v. plus 3-mal $500 \mathrm{mg}$ Metronidazol i.v. oder in der Eskalationsstufe 3-bis 4-mal 4,5g Piperacilin/Tazobactam i.v. oder 4-mal 500 mg Imipenem/Cilastatin i.v.) sowie die ultraschallgestützte Aspiration oder Drainage größerer Abszesse. Amöbenleberabszesse können durch Gabe von Metronidazol (3-mal $10 \mathrm{mg} / \mathrm{kg} \mathrm{KG}$ i.v. über 10 Tage) zur Einschmelzung gebracht werden und benötigen keine Drainage [68].

\section{Helicobacter-pylori-Infektion des Magens}

Helicobacter pylori ist der häufigste bakterielle Erreger des Menschen und Verursacher von chronischer Gastritis und Duodenitis mit Ausbildung von Ulzera. Aufgrund des deutlich erhöhten Risikos von Magenkarzinomen und MALT-Lymphomen bei chronischer Infektion wurde H. pylori als Karzinogen klassifiziert [69].

Therapie. Prinzipiell sollte eine H.-pylori-Infektion bei allen Patienten mit klinischen Symptomen mittels geeigneter Eradikationsschemata behandelt werden (s. „Eradikation von H. pylori-Schemata und Resistenzlage“ u. Tab.3).

Der als Karzinogen klassifizierte Helicobacter pylori sollte bei allen Patienten mit klinischer Symptomatik eradiziert werden.

\section{Akute Pankreatitis}

Die akute Pankreatitis ist primär keine Infektionskrankheit und verläuft initial fast immer abakteriell. Allerdings entwickelt fast ein Drittel aller Patienten mit akuter Pankreatitis sekundär Infektionen, die mit einer Erhöhung des Mortalitätsrisikos einhergehen [74]. Fieber, Leukozytose, Anstieg von C-reaktivem Protein (CRP) und abdominelle Schmerzen in der Frühphase sind Bestandteil des systemischen Entzündungsgeschehens, sind aber nicht beweisend für eine Infektion und benötigen daher keine Antibiotikatherapie [56].

Superinfektion von Nekrosen. Bei erneutem Anstieg der Entzündungsparameter oder Beschwerdeprogredienz nach bereits eingetretener Besserung der Symptomatik hingegen ist auf das Vorliegen einer Infektion zu achten. Typischerweise tritt diese als Superinfektion von nekrotischen Pankreasarealen auf. Für Imipenem/

\section{Eradikation von H. pylori}

\section{Schemata und Resistenzlage}

Während der letzten 2 Jahrzehnte waren die sog. französische bzw. bei Penicillinallergie die sog. italienische Tripeltherapie die Standardtherapien in der Behandlung von H.-pylori-Infektionen (Tab. 3) [70], mit Eradikationsraten von 70 - $80 \%$. Ansteigende Resistenzraten, vor allem gegen Clarithromycin, verkomplizieren die Therapie mittlerweile auch in unseren Breiten [71], sodass Standardtherapien nur noch für Regionen empfohlen werden, in denen die Resistenzrate gegenüber Clarithromycin unter $20 \%$ liegt (in den meisten Regionen Deutschlands aktuell ca. 10\%). Dabei ist zu bedenken, dass die Resistenzraten bei Personen aus dem südeuropäi- schen Raum (auch in der 2. Generation) und bei Migranten deutlich höher liegen können [72]. Mittel der Wahl wäre in einer derartigen Situation eine Quadrupeltherapie, bestehend aus Protonenpumpeninhibitor (PPI), Bismutsubzitrat, Tetracyclin und Metronidazol für 10 - 14 Tage als Erstlinientherapie [73]. Andere Schemata verwenden dieselben Antibiotika in anderer Reihenfolge (Tab. 3). Bei Versagen der Quadrupeltherapie wird eine ausreichend lang applizierte Kombinationstherapie aus PPI mit Amoxicillin und Levofloxacin (über 10 - 14 Tage) empfohlen. Weitere Eradikationsversuche sollten auf einer genauen Resistenztestung basieren [72].

\section{Tabelle 3}

Eradikationsschemata für die Behandlung der H.-pylori-Infektion [72, 73].

\begin{tabular}{|c|c|c|}
\hline Therapie & Antibiotika p.o. & Therapiedauer \\
\hline $\begin{array}{l}\text { Tripeltherapie } \\
\text { (französisch) }\end{array}$ & $\begin{array}{l}\text { PPI, } 500 \text { mg Clarithromycin, } \\
1000 \text { mg Amoxicillin, jeweils } 2 \times \text { tgl. }\end{array}$ & 10 - 14 Tage \\
\hline $\begin{array}{l}\text { Tripeltherapie } \\
\text { (italienisch) }^{1}\end{array}$ & $\begin{array}{l}\text { PPI, } 500 \mathrm{mg} \text { Clarithromycin, } 500 \mathrm{mg} \\
\text { Metronidazol, jeweils } 2 \times \text { tgl. }\end{array}$ & 10 - 14 Tage \\
\hline Quadrupeltherapie ${ }^{2}$ & $\begin{array}{l}\text { PPI } 2 \times \text { tgl.; Bismutsubzitrat, } 500 \text { mg } \\
\text { Tetracyclin, } 500 \text { mg Metronidazol, } \\
\text { jeweils } 4 \times \text { tgl. }\end{array}$ & 10 - 14 Tage \\
\hline \multirow[t]{2}{*}{ Sequenztherapie } & $\begin{array}{l}\text { PPI, } 1000 \mathrm{mg} \text { Amoxicillin, } \\
\text { jeweils } 2 \times \text { tgl. }\end{array}$ & Tag $1-5$ \\
\hline & $\begin{array}{l}\text { PPI, } 500 \text { mg Levofloxacin, } 500 \mathrm{mg} \\
\text { Metronidazol, jeweils } 2 \times \mathrm{tgl} .\end{array}$ & Tag 6-10 \\
\hline \multirow[t]{2}{*}{ Hybridtherapie } & $\begin{array}{l}\text { PPI, } 1000 \mathrm{mg} \text { Amoxicillin, } \\
\text { jeweils } 2 \times \text { tgl. }\end{array}$ & Tag 1-7 \\
\hline & $\begin{array}{l}\text { PPI, } 1000 \mathrm{mg} \text { Amoxicillin, } 500 \mathrm{mg} \\
\text { Clarithromycin, } 500 \mathrm{mg} \text { Metroni- } \\
\text { dazol, jeweils } 2 \times \text { tgl. }\end{array}$ & Tag $8-14$ \\
\hline
\end{tabular}


Cilastatin wurde in einer Cochrane-Analyse eine signifikante Reduktion der Rate an Neuinfektionen von Pankreasnekrosen gefunden [75], allerdings ohne signifikante Verringerung der Mortalitätsrate. Die Autoren weisen darauf hin, dass die klinische Evidenz aufgrund unter-powerter Studien als gering einzuschätzen ist [75]. US-amerikanische Behandlungsrichtlinien empfehlen eine antibiotische Therapie, wenn mehr als 30\% des Pankreas in der CT Nekrosezonen aufweisen [74].

Eine generelle prophylaktische Gabe von Antibiotika bei akuter Pankreatitis wird nicht empfohlen, da diese keine Überlebensvorteile bietet, aber die Selektion von resistenten Erregerätiologien begünstigt.

\section{Komplikationen der chronischen Pankreatitis}

Auch bei chronischer Pankreatitis besteht keine Indikation für eine generelle antibiotische Prophylaxe bzw. Metaphylaxe. Bei der häufigen Pseudozystenbildung (20-40\% der Fälle) ist allerdings die Gefahr einer bakteriellen Superinfektion signifikant erhöht. Infizierte Pseudozysten sollten interventionell (vorzugsweise endosonografisch) oder operativ drainiert werden [76] und bedürfen einer gezielten antibiotischen Behandlung.

\section{Kernaussagen}

Infektiöse Durchfallerkrankungen

- Die symptomatische Therapie steht bei der Behandlung von infektiös bedingten Durchfallerkrankungen im Vordergrund. Der Nutzen einer empirischen Antibiotikatherapie ist umstritten.

- Situationen, die eine gezielte Erregerdiagnostik und evtl. auch spezifische Therapie erfordern, sind: schweres Krankheitsbild, prolongierte Diarrhö, Zeichen einer Organinvasivität des Erregers, vermuteter Ausbruch, Immunsuppression und signifikante Komorbidität.

- Die gezielte antimikrobielle Therapie von schweren infektiös bedingten Durchfallerkrankungen setzt eine differenzierte mikrobiologische Stuhluntersuchung voraus.
- Antibiotikaresistenzen - gerade bei importierten Fällen (vor allem aus Indien) - sind in zunehmendem Maße zu berücksichtigen.

\section{Andere bakterielle Infektionen}

- Anders sieht es bei bakteriell bedingten Infektionen von Magen, Leber, Gallenwegen oder Peritonealraum aus. Hier stellt die in der Regel empirisch verabreichte Antibiotikatherapie eine wichtige Säule im Behandlungskonzept dar.

- Neben klaren Entscheidungen für eine operative Therapie, bei der die Antibiotikatherapie nur adjuvanten Charakter besitzt, sind die Kenntnis und Wertung von Erregereigenschaften wie Resistenzen und wirtseigenen Faktoren wichtig für die optimale und sichere Auswahl geeigneter Therapeutika.
Interessenkonflikte: Es bestehen keine Interessenkonflikte.

\section{Über den Autor}

\section{Christoph Lübbert}

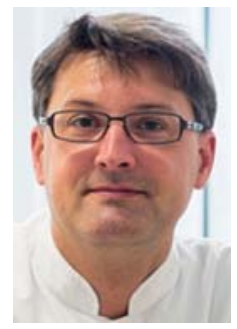

Priv.-Doz. Dr. med. habil. Jahrgang 1971. Medizinstudium an den Universitäten Kiel, Zürich (Schweiz) und Durban (Südafrika). Promotion 1999. Klinische Ausbildung zum Internisten, Gastroenterologen und Infektiologen mit Zusatzausbildung in der Tropenmedizin (D.T.M.\&H., Tropeninstitut Liverpool) von 2000-2008 in Ratze-

burg, Leipzig, Liverpool (Großbritannien) und Halle/Saale. 2008-2012 Tätigkeit als Oberarzt am Universitätsklinikum Halle/Saale mit den Schwerpunkten Endoskopie und Klinische Infektiologie. 2012 Übernahme der Fachbereichsleitung Infektions- und Tropenmedizin innerhalb der Klinik für Gastroenterologie und Rheumatologie des Universitätsklinikums Leipzig. Klinische Forschungsinteressen: Multiresistente Erreger (MRE) und ihre Verbreitung, Clostridium-difficile-Infektionen (CDI), Antibiotic Stewardship (ABS) sowie die klinische Bedeutung von mikrobiellen Besiedelungen in der Gastroenterologie/ Endoskopie.

\section{Korrespondenzadresse}

Priv.-Doz. Dr. med. Christoph Lübbert

Leiter des Fachbereichs Infektions- und Tropenmedizin Klinik und Poliklinik für Gastroenterologie und

Rheumatologie

Universitätsklinikum Leipzig

Liebigstraße 20

04103 Leipzig

E-Mail: christoph.luebbert@medizin.uni-leipzig.de

\section{Literatur}

1 Lübbert C, Weis S. Medikamentöse Therapie der infektiösen Diarrhoe - Teil 1: Akute Diarrhoe. Internist 2013; 54: 1383 1392

2 World Health Organization (WHO). Diarrhoeal disease. Im Internet: http://www.who.int/mediacentre/factsheets/ fs330/en/ (Stand: 9.7.2015) 
3 Theuretzbacher U. Accelerating resistance, inadequate antibacterial drug pipelines and international responses. Int J Antimicrob Agents 2012; 39: 295-299

4 Sullivan A, Edlund C, Nord CE. Effect of antimicrobial agents on the ecological balance of human microflora. Lancet Infect Dis 2001; 1: 101-114

5 Thielman NM, Guerrant Rl. Clinical practice. Acute infectious diarrhea. N Engl J Med 2004; 350: 38 - 47

6 Wistrom J, Jertborn M, Ekwall E et al. Empiric treatment of acute diarrheal disease with norfloxacinA randomized, placebo-controlled study. Swedish Study Group. Ann Intern Med 1992; 117: $202-208$

7 Adachi JA, Ericsson CD, Jiang ZD et al. Azithromycin found to be comparable to levofloxacin for the treatment of US travelers with acute diarrhea acquired in Mexico. Clin Infect Dis 2003; 37: $1165-1171$

8 Hong KS, Kim JS. Rifaximin for the treatment of acute infectious diarrhea. Therap Adv Gastroenterol 2011; 4: 227 235

9 Taneja N. Changing epidemiology of shigellosis and emergence of ciprofloxacin-resistant Shigellae in India. J Clin Microbiol 2007; 45: 678-679

10 Vogelmann R, Ebert M. Multiresistente Erreger in der Gastroenterologie - Folgen für die Wahl des Antibiotikums. Gastroenterologe 2014; 9: 565-578

11 Lübbert C, Rodloff AC, Laudi S et al. Lessons learned from excess mortality associated with Klebsiella pneumoniae carbapenemase-2-producing $\mathrm{K}$. pneumoniae in liver transplant recipients. Liver Transpl 2014; 20: 736 - 738

12 Adriaenssens N, Coenen S, Versporten A et al. European Surveillance of Antimicrobial Consumption (ESAC): outpatient antibiotic use in Europe (1997-2009). J Antimicrob Chemother 2011; 66: vi3 - vi12

13 Robert-Koch-Institut (RKI). Antibiotika Resistenz Surveillance (ARS). Im Internet: http://ars.rki.de/ (Stand: 9.7.2015)

14 Onwuezobe IA, Oshun PO, Odigwe CC. Antimicrobials for treating symptomatic non-typhoidal Salmonella infection. Cochrane Database Syst Rev 2012; 11: CD001167

15 Robert-Koch-Institut (RKI). Salmonellose. Ratgeber für Ärzte. Im Internet: http://www.rki.de/DE/Content/Infekt/EpidBull/ Merkblaetter/Ratgeber_Salmonellose.html (Stand: 9.7.2015)

16 Robert-Koch-Institut (RKI). Shigellose. Ratgeber für Ärzte. Im Internet: http://www.rki.de/DE/Content/Infekt/EpidBull/ Merkblaetter/Ratgeber_Shigellose.html (Stand: 9.7.2015)

17 Christopher PR, David KV, John SM et al. Antibiotic therapy for Shigella dysentery. Cochrane Database Syst Rev 2010; 8: CD006784

18 Robert-Koch-Institut (RKI). Campylobacter-Infektionen. Ratgeber für Ärzte. Im Internet: http://www.rki.de/DE/Content/ Infekt/EpidBull/Merkblaetter/Ratgeber_Campylobacter.html (Stand: 9.7.2015)

19 Kirkpatrick BD, Tribble DR. Update on human Campylobacter jejuni infections. Curr Opin Gastroenterol 2010; 27: 1 - 7

20 Lehtopolku M, Kotilainen P, Haanpera-Heikkinen M et al. Ribosomal mutations as the main cause of macrolide resistance in Campylobacter jejuni and Campylobacter coli. Antimicrob Agents Chemother 2011; 55: 5939-5941

21 Buchholz U, Bernard H, Werber D et al. German outbreak of Escherichia coli O104:H4 associated with sprouts. N Engl ] Med 2011; 365: $1763-1770$
22 Nitschke M, Sayk F, Hartel C et al. Association between azithromycin therapy and duration of bacterial shedding among patients with Shiga toxin-producing enteroaggregative Escherichia coli O104:H4. JAMA 2012; 307: 1046-1052

23 Harris JB, Larocque RC, Qadri F et al. Cholera. Lancet 2012; 379: $2466-2476$

24 Lübbert C, John E, von Müller L. Clostridium difficile-Infection (CDI): Guideline-based diagnosis and treatment. Dtsch Ärztebl Int 2014; 111: $723-731$

25 Burckhardt F, Friedrich A, Beier D et al. Clostridium difficile surveillance trends, Saxony, Germany. Emerg Infect Dis 2008; 14: $691-692$

26 Hensgens MP, Goorhuis A, Dekkers OM et al. Time interval of increased risk for Clostridium difficile infection after exposure to antibiotics. J Antimicrob Chemother 2011; 67: $742-748$

27 Zar FA, Bakkanagari SR, Moorthi KM et al. A comparison of vancomycin and metronidazole for the treatment of Clostridium difficile-associated diarrhea, stratified by disease severity. Clin Infect Dis 2007; 45: $302-307$

28 Bauer MP, Kuijper EJ, Van Dissel JT et al. European Society of Clinical Microbiology and Infectious Diseases (ESCMID): treatment guidance document for Clostridium difficile infection (CDI). Clin Microbiol Infect 2009; 15: 1067 - 1079

29 Debast SB, Bauer MP, Kuijper EJ et al. European Society of Clinical Microbiology and Infectious Diseases: update of the treatment guidance document for Clostridium difficile infection. Clin Microbiol Infect 2014; $20: 021-26$

30 Gerding DN, Muto CA, Owens RCJr. Treatment of Clostridium difficile infection. Clin Infect Dis 2008; $46: 0132$ - 542

31 Weis S, John E, Lippmann $\mathrm{N}$ et al. Clostridium difficile - ein Thema nur für den Internisten? Zentralbl Chir 2014; 139: $460-468$

32 Pillai A, Nelson R. Probiotics for treatment of Clostridium difficile-associated colitis in adults. Cochrane Database Syst Rev 2008; 1: CD004611

33 Johnston BC, Ma SS, Goldenberg JZ et al. Probiotics for the prevention of Clostridium difficile-associated diarrhea: a systematic review and meta-analysis. Ann Intern Med 2013; 157: $878-888$

34 Louie TJ, Miller MA, Mullane KM et al. Fidaxomicin versus vancomycin for Clostridium difficile infection. N Engl J Med 2011; 364: $422-431$

35 Van Nood E, Vrieze A, Nieuwdorp M et al. Duodenal infusion of donor feces for recurrent Clostridium difficile. N Engl J Med 2013; 368: 407-415

36 Lowy I, Molrine DC, Leav BA et al. Treatment with monoclonal antibodies against Clostridium difficile toxins. N Engl J Med 2010; 362: 197-205

37 Sandhu DK, Surawicz C. Update on chronic diarrhea: a runthrough for the clinician. Curr Gastroenterol Rep 2012; 14: $421-427$

38 Lübbert C, Weis S. Medikamentöse Therapie der infektiösen Diarrhoe - Teil 2: Chronische Diarrhoe. Internist 2013; 54 : $1513-1519$

39 Lalle M. Giardiasis in the post genomic era: treatment, drug resistance and novel therapeutic perspectives. Infect Disord Drug Targets 2010; 10: 283-294

40 Granados CE, Reveiz L, Uribe LG et al. Drugs for treating giardiasis. Cochrane Database Syst Rev 2012; 12: CD007787 
41 Stanley SLJr. Amoebiasis. Lancet 2003; 361: 1025-1034

42 Haque R, Huston CD, Hughes M et al. Amebiasis. N Engl J Med 2003; 348: 1565 - 1573

43 Marth T, Raoult D. Whipple‘s disease. Lancet 2003; 361: 239246

44 Feasey NA, Healey P, Gordon MA. Review article: the aetiology, investigation and management of diarrhoea in the HIVpositive patient. Aliment Pharmacol Ther 2011; 34: 587-603

45 Allers K, Schneider T. Gastrointestinale Manifestationen der HIV-Infektion. Gastroenterologie up2date 2012; 8: 219-234

46 Nissapatorn V, Sawangjaroen N. Parasitic infections in HIV infected individuals: diagnostic \& therapeutic challenges. Indian J Med Res 2011; 134: 878-897

47 Abubakar I, Aliyu SH, Arumugam C et al. Prevention and treatment of cryptosporidiosis in immunocompromised patients. Cochrane Database Syst Rev 2007; 1: CD004932

48 Wright SG. Protozoan infections of the gastrointestinal tract. Infect Dis Clin North Am 2012; 26: 323-239

49 Lutz P, Nischalke HD, Strassburg CP et al. Spontaneous bacterial peritonitis: The clinical challenge of a leaky gut and a cirrhotic liver. World J Hepatol 2015; 7: 304 - 314

50 Jalan R, Fernandez J, Wiest R et al. Bacterial infections in cirrhosis: a position statement based on the EASL Special Conference 2013. J Hepatol 2014; 60: 1310-1324

51 Navasa M, Follo A, Llovet JM et al. Randomized, comparative study of oral ofloxacin versus intravenous cefotaxime in spontaneous bacterial peritonitis. Gastroenterology 1996 111: $1011-1017$

52 Chavez-Tapia NC, Soares-Weiser K, Brezis M et al. Antibiotics for spontaneous bacterial peritonitis in cirrhotic patients. Cochrane Database Syst Rev 2009; 1: CD002232

53 Fernández J, Navasa M, Planas R et al. Primary prophylaxis of spontaneous bacterial peritonitis delays hepatorenal syndrome and improves survival in cirrhosis. Gastroenterology 2007; 133: $818-824$

54 Ginés P, Rimola A, Planas R et al. Norfloxacin prevents spontaneous bacterial peritonitis recurrence in cirrhosis: results of a double-blind, placebo-controlled trial. Hepatology 1990; 12: $716-724$

55 Fernández J, Acevedo J, Castro $\mathrm{M}$ et al. Prevalence and risk factors of infections by multiresistant bacteria in cirrhosis: a prospective study. Hepatology 2012; 55: 1551 - 1561

56 Bodmann KF. Expertenkommission der Infektliga. Komplizierte intraabdominelle Infektionen: Erreger, Resistenzen. Chirurg 2010; 81: 38 - 49

57 Doklestić SK, Bajec DD, Djukić RV et al. Secondary peritonitis evaluation of 204 cases and literature review. J Med Life 2014; 7: $132-138$

58 Solomkin JS, Ristagno RL, Das AF et al. Source control review in clinical trials of anti-infective agents in complicated intraabdominal infections. Clin Infect Dis 2013; 56: 1765-1773

59 Peery AF, Dellon ES, Lund J et al. Burden of gastrointestinal disease in the United States: 2012 update. Gastroenterology 2012; 143: $1179-1187$
60 Chabok A, Pahlmann L, Hjern F et al. Randomized clinical trial of antibiotics in acute uncomplicated diverticulitis. Br J Surg 2012; 99: $532-539$

61 Fuks D, Cossé C, Régimbeau JM. Antibiotic therapy in acute calculous cholecystitis. J Visc Surg 2013; 150: 3-8

62 Ambe P, Weber SA, Christ H et al. Cholecystectomy for acute cholecystitis. How time-critical are the so called "golden 72 hours"? Or better "golden 24 hours" and "silver 25-72 hour"? A case control study World J Emerg Surg 2014; 9: 60

63 Nishino T, Hamano T, Mitsunaga Y et al. Clinical evaluation of the Tokyo Guidelines 2013 for severity assessment of acute cholangitis. J Hepatobiliary Pancreat Sci 2014; 21: 841 - 849

64 Regimbeau JM, Fuks D, Pautrat K et al. Effect of postoperative antibiotic administration on postoperative infection following cholecystectomy for acute calculous cholecystitis: a randomized clinical trial. JAMA 2014; 312: 145 - 154

65 Mazeh H, Mizrahi I, Dior U et al. Role of antibiotic therapy in mild acute calculus cholecystitis: a prospective randomized controlled trial. World J Surg 2012; 36: 1750 - 1759

66 Mosler P. Management of acute cholangitis. Gastroenterol Hepatol (NY) 2011; 7: 121-123

67 Tanaka A, Takada T, Kawarada Y et al. Antimicrobial therapy for acute cholangitis: Tokyo Guidelines. J Hepatobiliary Pancreat Surg 2007; 14: 59-67

68 Lübbert C, Wiegand J, Karlas T. Therapy of liver abscesses. Viszeralmedizin 2014; 30: 334 - 341

69 Malfertheiner P, Chan FK, McColl KE. Peptic ulcer disease. Lancet 2009; 374: 1449-1461

70 Papastergiou V, Georgopoulos SD, Karatapanis S. Treatment of Helicobacter pylori infection: Past, present and future. World J Gastrointest Pathophysiol 2014; 5: 392 - 399

71 Smith SM, O’Morain C, McNamara D. Antimicrobial susceptibility testing for Helicobacter pylori in times of increasing antibiotic resistance. World J Gastroenterol 2014; 20: 9912 9921

72 Scherübl H, Fischbach W, Glocker E et al. [What is new in treating Helicobacter pylori infection?]. Dtsch Med Wochenschr 2015; 140: 277 - 280

73 Malfertheiner P, Megraud F, O'Morain CA et al. Management of Helicobacter pylori infection - the Maastricht IV/Florence Consensus Report. Gut 2012; 61: 646-664

74 Banks PA, Freeman ML. Practice Parameters Committee of the American College of Gastroenterology. Practice guidelines in acute pancreatitis. Am J Gastroenterol 2006; 101: 23792400

75 Villatoro E, Mulla M, Larvin M. Antibiotic therapy for prophylaxis against infection of pancreatic necrosis in acute pancreatitis. Cochrane Database Syst Rev 2010; 5: CD002941

76 Hoffmeister A, Mayerle ], Beglinger C et al. [S3-Consensus guidelines on definition, etiology, diagnosis and medical, endoscopic and surgical management of chronic pancreatitis German Society of Digestive and Metabolic Diseases (DGVS)]. Z Gastroenterol 2012; 50: 1176-1224 


\section{CME॰thieme.de}

\section{CME-Fragen}

\section{CME-Teilnahme}

- Viel Erfolg bei lhrer CME-Teilnahme unter http://cme.thieme.de

- Diese Fortbildungseinheit ist 12 Monate online für eine CME-Teilnahme verfügbar.

- Sollten Sie Fragen zur Online-Teilnahme haben, unter http://cme.thieme.de/hilfe finden Sie eine ausführliche Anleitung.

Welches der folgenden genannten Antibiotika spielt bei der empirischen Antibiotikatherapie einer infektiösen Diarrhö keine Rolle?
A Ciprofloxacin
B Metronidazol
C Levofloxacin
D Rifaximin
E Azithromycin

2 Welche Aussage zur Clostridium-difficile-Infektion (CDI) ist richtig?

A Die i.v. Verabreichung monoklonaler Antikörper gegen Toxin A und B ist keine therapeutische Option zur Rezidivprophylaxe.

B Antibiotikaeinnahme schützt vor dem Erleiden einer CDI.

C Mikrobiomtransfer erbrachte sehr gute Behandlungsergebnisse bei mehrfachem CDI-Rezidiv.

D Die Therapie mit Fidaxomicin weist deutlich höhere Rezidivraten auf als die Therapie mit Vancomycin.

E Bei schweren Verläufen war in Studien die Therapie mit Metronidazol der Vancomycin-Therapie überlegen.

3 Welche Aussage zur Shigellose ist richtig?

A Eine Resistenztestung ist bei Shigellen nicht erforderlich.

B Gegenüber Fluorchinolonen sind bisher keine Resistenzen bei Shigellen bekannt.

C Azithromycin wird zur Behandlung der Shigellose prinzipiell empfohlen.

D Motilitätshemmer sind eine wichtige Therapiesäule in der Behandlung der Shigellose.

E Eine blutig-schleimige Diarrhö kommt bei der Shigellose im Gegensatz zur Salmonellose nicht vor.

4 Welche Aussage zur Divertikulitis ist falsch?

A Nach neuerer Studienlage ist eine Antibiotikatherapie bei unkomplizierter Divertikulitis nicht notwendig.

B Bei Vorhandensein einer Peridivertikulitis und bei Nachweis eines phlegmonösen Entzündungsmusters wird eine antibiotische Therapie durchgeführt.

C Cefotaxim ist für die empirische Therapie der Divertikulitis nicht geeignet.

D Resistente Enterobakterien und Enterokokken können ein Wirkversagen der empirischen Antibiotikatherapie zur Folge haben.

E Komplikationen wie eine Perforation und oder Abszedierungen erfordern eine operative Versorgung.

5 Welche Aussage zur Helicobacter-pylori-(H.p.-)Infektion des Magens ist richtig?

A Bei Penicillinallergie soll die französische Tripeltherapie (mit Amoxicillin) zum Einsatz kommen.

B Eine Eradikationstherapie ist nur bei Vorliegen eines MALT-Lymphoms erforderlich.

C Primäre Therapie ist die Gabe von mindestens zwei H.p.-wirksamen Antibiotika, kombiniert mit einem Protonenpumpenhemmer (PPI).

D Eine H.p.-Infektion führt immer zu einem Ulcus ventriculi.

E Resistenzen von $\mathrm{H}$. pylori gegen Clarithromycin sind bisher noch nicht aufgetreten. 


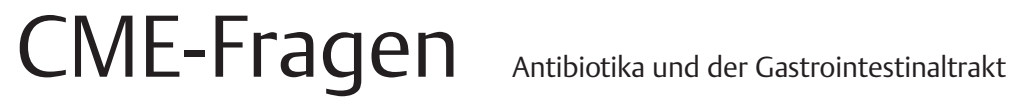

6 Welche Aussage zur Salmonellen-Enteritis ist richtig?

A Resistenzbildung spielt bei Salmonellen keine Rolle.

B Schwere Verläufe und Komplikationen treten vor allem bei abwehrgeschwächten Patienten auf.

C Eine konsequente antibiotische Therapie beugt der Entwicklung der Dauerausscheidung vor.

D Wichtigster Erreger in Deutschland ist das Serovar Typhi.

E Die Inzidenz von Salmonellosen liegt in Deutschland deutlich über der Inzidenz von Campylobacter-Infektionen.

7 Welche Aussage zur HIV-Infektion bzw. AIDS ist richtig?

A Die hochaktive antiretrovirale Therapie (HAART) hat keinen Einfluss auf die Ausheilung von chronischen Darminfektionen bei HIV, wie z. B. die Kryptosporidiose.

B Der Nachweis von Kryptosporidien oder Mikrosporidien bei HIV im Stuhl ist unkompliziert anhand von Schnelltests möglich.

C Gewichtsverlust und Durchfall sind keine typischen Symptome von AIDS.

D Die medikamentöse Behandlung einer HIV-Infektion ist stets eine Kombinationstherapie aus mehreren Medikamenten.

E Gastrointestinale Probleme durch Kryptosporidiose oder Mikrosporidiose spielen bei der HIV-Infektion heutzutage keine Rolle mehr.

8 Welches Symptom gehört nicht zur sog. Reynold-Pentade bei akuter Cholangitis?
A Fieber
B Oberbauchschmerz
C Hypotension
D Verwirrtheitszustände
E verminderte Darmgeräusche

9 Welches Medikament gehört nicht zur Quadrupeltherapie der H.-pylori-Infektion?
A Bismutsalz
B Clarithromycin
C Protonenpumpenhemmer
D Tetracyclin
E Metronidazol

10 Welche Aussage zur sekundären bakteriellen Peritonitis ist falsch?

A Es handelt sich um eine Infektion der Bauchhöhle mit einem chirurgisch sanierbaren Fokus.

B Es liegt typischerweise eine monobakterielle Infektion des Bauchraums vor.

C Häufige Auslöser sind Ulcera duodeni und ventriculi, Appendizitis oder Divertikulitis.

D Die Therapie besteht aus der chirurgischen Sanierung des Fokus und der Gabe von Breitspektrum-Antibiotika.

E Die Mortalitätsrate ist trotz adäquater chirurgischer und antibiotischer Therapie hoch. 